\title{
Testing soil fertility of Prehispanic terraces at Viejo Sangayaico in the upper Ica catchment of south-central highland Peru
}

\author{
William P. Nanavati ${ }^{a}$, Charles French ${ }^{b, *}$, Kevin Lane ${ }^{c}$, Oliver Huaman Oros $^{d}$, David Beresford-Jones ${ }^{c}$ \\ a Department of Earth Sciences, Montana State University, Traphagen Hall, Bozeman, MT 59717, USA \\ ${ }^{\mathrm{b}}$ Department of Archaeology and Anthropology, University of Cambridge, Downing Street, Cambridge CB2 3DZ, UK \\ c McDonald Institute for Archaeological Research, University of Cambridge, Downing Site, Cambridge CB2 3ER, UK \\ d Calle Germán Amézaga N 375, Edificio Jorge Basadre, Universidad Nacional Mayor de San Marcos, Ciudad Universitaria, Lima 1, Peru
}

\section{A R T I C L E I N F O}

\section{Article history:}

Received 27 November 2015

Received in revised form 23 February 2016

Accepted 7 March 2016

Available online xxxx

\section{Keywords:}

Argillic tethering

Geo-chemistry

Micromorphology

Soil fertility

Terraces

\begin{abstract}
A B S T R A C T
This study presents a pilot geoarchaeological investigation of terraced agricultural systems near San Francisco de Sangayaico, in the upper Ica catchment of the Southern Peruvian Andes. It aims to assess the evidence for soil fertility associated with agricultural strategies practised throughout the Prehispanic, Spanish colonial and modern occupations in this region. A series of twenty-two test pits were hand excavated through two terraced field systems, and sampled to examine the changes in soil physical and chemical characteristics down-profile and downslope.

This study provides the first geoarchaeological analyses of the agrarian soil system surrounding Viejo Sangayaico in the upper Ica catchment. Results demonstrate that the soil system was much modified prior to the creation of the terrace systems, probably about 900 years ago. This system was characterised by a weakly acidic to slightly calcareous $\mathrm{pH}$, a consistent but low electrical conductivity, reasonable-but-variable phosphorus content, and a loamy soil texture with a component of weathered volcanic tonalite parent material. The shallow terrace soil build-up on the slopes investigated indicates that slope modification was as minimal as possible. Moreover, the relatively low frequencies of organic material and phosphorus suggest that the terraces were not heavily fertilised in the past, making the stability and management of the nutrient-rich topsoil vital.

The results of these excavations and soil fertility analyses are situated within the context of the wider Andean ethno-historic and the archaeological record to address questions regarding how the terraces were built and maintained over time. Agricultural terraces undoubtedly mitigated the effects of slope erosion associated with cultivation. But, the terrace soil features observed at Sangayaico do not appear to be the same as those documented in other geoarchaeological studies of Andean terrace systems. These contrasts may be accounted for by a combination of differing geological substrate and hydrological conditions, as well as variable trajectories in past soil development, erosion factors, manuring/field management practises and crop selection.
\end{abstract}

(C) 2016 Published by Elsevier B.V.

\section{Introduction}

In this study the soil fertility of two Andean terrace systems and their associated buried soils adjacent to the archaeological site of Viejo Sangayaico (or SAN1) is assessed (Fig. 1). It is set within the Quebrada Marcaccarancca of the highland Olaya tributary of the Río Ica in the south-central Andes of Peru. The site and its environs including ancillary settlements cover c. $30 \mathrm{~km}^{2}$ situated between 2800 and 4200 m.a.s.l., and were ethnically part of the Late Prehispanic Chocorvos 'nation' (Puglar, 1946). This site is dated to Late Intermediate, Inca and Spanish colonial periods with a range of radiocarbon dates from cal AD $1122 \pm$ 81 (952 \pm 27 BP; OxA-30914-6) to cal AD $1527 \pm 88$ (362 \pm 27 BP; OxA-30930-1). It is located at the transition between the low-lying

\footnotetext{
* Corresponding author.

E-mail address: caif2@cam.ac.uk (C. French).
}

agricultural quechua ecozone (or irrigated, cultivated terrace zone at 2300-3500 m.a.s.l.) and the increasingly agro-pastoralist suni (or dry field agricultural zone at 3500-3800 m.a.s.l.) and puna ecozones (or upland pasture zone at 3800-4800 m.a.s.l.) (Covey, 2006; D'Altroy, 2003; Pulgar Vidal, 1946). The area exhibits significant technological investment in terracing and irrigation canals for agriculture on the mountain slopes of the quechua zone, and high altitude check dams in the high puna zone geared towards the creation of good pasturage. Viejo Sangayaico appears to have been situated so as to control access to and from these ecozones and across the northern Pisco Valley, an important hub of Chorcorvos (AD 1000-1450) and later Inca (AD 14501532) and Spanish (post-AD 1532) occupations (Bueno Mendoza, 2003; Chauca Iparraguirre and Lane, 2015; Huaman Oros and Lane, 2014; Lane et al., 2015). Although no direct dating evidence was retrieved from the palaeosols and terrace systems at Sangayaico, it is highly likely that the construction and use of the extensive bench terracing 


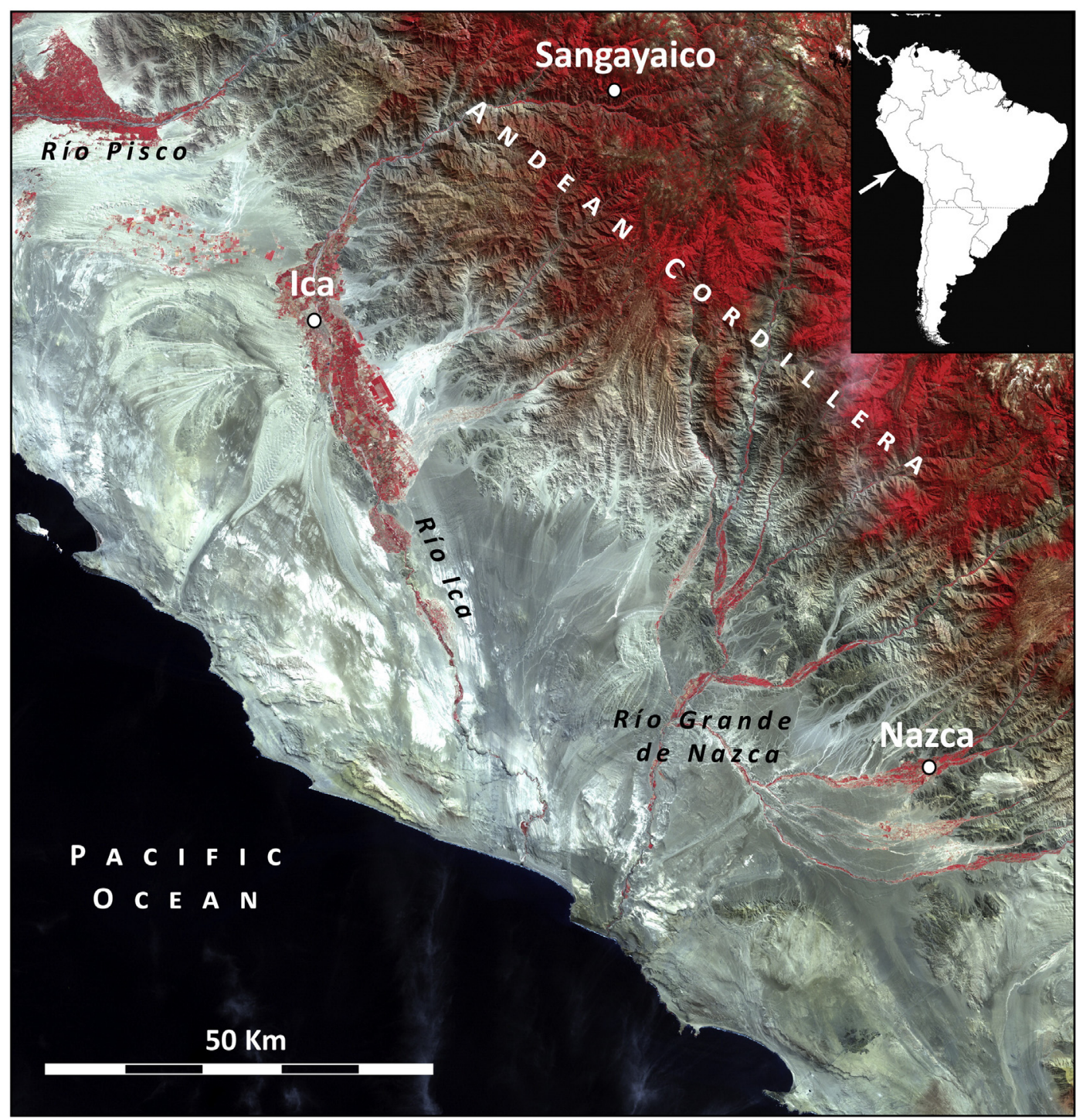

Fig. 1. Location map of Sangayaico in the upper Ica valley of southern Peru. D. Beresford-Jones, based on LANDSAT 7 ETM + 2000, USGS.

found in the area below c. 3500 m.a.s.l. relates to the same periods as the adjacent occupations recorded and radiometrically dated. Situated in an area rich in agricultural terracing, both abandoned and in use, this paper presents a preliminary assessment of soil fertility of part of the terrace system around the Sangayaico sites, as well as providing important comparative information concerning the creation and maintenance of terraces in the Andean highlands.

Geologically, the area is composed primarily of tonalite parent material originating from volcanic activity that occurred during the Cerozoic-Quaternary transition (Palacios Moncayo, 1994). Tonalite is primarily composed of andesine, biotite, hornblende, quartz, and orthoclase minerals (Nettleton et al., 1970). The weathering of biotite in tonalite produces clay particles such as kaolinite and vermiculite, as well as silicaceous mica, and hornblende and quartz weathering produces sand, silt and clay particles (ibid., 1970). It is these weathered mineral constituents that define the substrate characteristics of the landscape surrounding Sangayaico. Stone and coarse-fine sand-size fragments of this tonalite parent material are found throughout the soil materials composing the terrace systems. The regional soils developed on this parent rock range from luvisols with evidence of clay migration to weakly developed cambisols, with leptosols on the steeper, rockier slopes (Gardi et al., 2015; WRB, 2014).

Soil fertility analyses are a crucial tool in the characterisation of past and present agro-ecosystems (Sandor et al., 2007). Given the scale of human impact on the Andes (Denevan, 1992; Lentz, 2000), it is surprising that so few geoarchaeological studies of terrace systems exist in this geographical area, especially given the large amount of work that has been done on terraces more generally in Peru (i.e. Branch et al., 2007; de la Torre and Burga, 1986; Farrington, 1980; Gelles, 2000; Kemp et al., 2006; Kendall and Chepstow-Lusty, 2006; Kosok, 1965; Mitchell and Guillet, 1994; Schjellerup, 1986; Trawick, 2003; Treacy, 1994; Williams, 2006). This study goes some way to rectifying this gap in our knowledge and provides comparative soil fertility analyses across several agricultural systems, thus providing important insights on the interpretation of past agricultural strategies and other socio-cultural practises in the region (Goodman-Elgar, 2009; Sandor et al., 2007; Wells, 2006).

The many previous studies of terraces elsewhere in the Americas have suggested that there is firm evidence for terracing in the Preclassic Mayan period c. 2000 years ago, reaching a peak in the Late Classic period about 1300 years ago (Beach et al., 2015; Bonavia, 1967-1968; 
Denevan, 2001; Donkin, 1979). Terraces fulfil four main functions: providing a platform for a deep soil matrix which facilitates cultivation, control of erosion, the creation of a sustainable micro-climate and control of humidity (Treacy, 1994), as well as enhanced soil moisture and organic matter contents. The classic image of Andean terracing and those found in the study area is that of bench terraces, variously known as andenes, patasi, bancales and takhanes (Denevan, 2001). Around Sangayaico, the terrace walls are constructed using dry-walling known locally as pirca masonry (Fig. 3). Although few of these terraces have been excavated, those that have such as in the Paca, Cuzco, Colca and Chicha-Soras valleys were similarly built with either a single or double course of pirca dry-stone masonry walls (Bonavia, 1967-1968; Brooks, 1998; Denevan, 2001; Donkin, 1979; Goodman-Elgar, 2002; Kemp et al., 2006; Londoño, 2008; Schjellerup, 1986; Treacy, 1994).

Previous geoarchaeological studies have observed a number of important characteristics of Andean terrace systems. These include the burying of original agricultural horizons during terrace construction, the preferential construction of terraces above argillic horizons - referred to as tethering (Homburg et al., 2005), the application of fertiliser and the use of seasonal burning (Table 1). In particular, the tethering of agricultural terraces to argillic horizons is known from the Colca Canyon, and the Paca and Chicha-Soras valleys in Peru (Goodman-Elgar, 2008; Kemp et al., 2006; Sandor and Eash, 1991, 1995). Their creation and maintenance result in the formation of anthropogenic topsoil covering the original agricultural horizons which have been profoundly affected by physical, hydrological and geochemical alterations (Bryant and Galbraith, 2010). This may result in elevated phosphorous and organic matter levels, a decreased $\mathrm{pH}$ and increased concentrations of illuvial silt, clay, and organic matter (Goodman-Elgar, 2008; Sandor and Eash, 1991, 1995). Andean studies by most of these same authors also note the presence of archaeological artefacts throughout the terrace sequence, thus suggesting the repeated application of household waste as fertiliser through middening. There may also have been corralling of animals to specific fields, most likely llamas or alpacas, and/or the collection and direct application of manure from separate corrals. These techniques would have supplemented the organic matter status of the field, increasing the nutrient content as well as the water holding capacity of the soil, thereby enhancing the productivity of the landscape (Sandor and Eash, 1991, 1995). In addition, there may be spikes of immobile phosphorous (Holliday and Gartner, 2007; Sandor and Eash, 1995) and decreases in $\mathrm{pH}$ (to weakly acidic conditions) that were counteracted by seasonal burning leading to the increased availability of phosphorus (P), potassium (K) and carbonates. Regular burning would have also helped to remove deleterious micro-flora and microfauna whilst depositing nutrient rich ash (Thomaz et al., 2014).

According to local Sangayaiqueño farmers, once the growing season was over animals grazed on either the remains of the harvest or on the specially grown alfalfa (Medicago sativa), and then the terrace fields were regularly burnt off. Growing seasons are short and are followed by long fallow seasons of one to five years. These practises allow
Sangayaiqueño farmers to maintain a productive landscape without the use of artificial fertilisers.

At Sangayaico, two associated terrace systems were examined by targeted test pit excavations to investigate changes in soil physical and chemical characteristics, both down-profile and downslope. A total of 22 test pits were hand excavated across these terrace systems (Figs. 2-5), described and sampled for physical, chemical and micromorphological analyses.

The results of this pilot study are then compared to other investigations of terrace systems in the wider literature in order to address three main questions:

1. Is there evidence for buried agricultural horizons?

2. What past agricultural strategies (i.e. tethering to argillic horizon, fertiliser use, etc.) are evident in the terrace systems associated to the Sangayaico site?

3. How have past and present agricultural strategies affected soil fertility?

\section{Survey and laboratory methodology}

In the landscape around Viejo Sangayaico two terrace systems (Fig. 2, A/red and B/blue) were selected on the basis of their proximity to the SAN1 site and the apparent preservation of the terrace architecture. Terrace system A was situated immediately downslope of SAN1 and covered roughly $6 \mathrm{~km}^{2}$, ranging from an elevation of 35853625 m.a.s.l., and had been left fallow for the past three to five years. Below Terrace system A, Terrace system B was in use at the time of excavation which limited excavation to three test pits. This system covers roughly $12 \mathrm{~km}^{2}$, ranging in elevation from 3532 to 3577 m.a.s.l. Nineteen test pits were excavated (either $50 \mathrm{~cm}$ or $100 \mathrm{~cm}$ square) in the upper slope area (Terrace System A: TP1-4 \& 6-20) along three parallel transects perpendicular to the slope to the top of weathered tonalite bedrock, and a further three test pits were excavated in a lower terrace system in a single transect (Terrace System B: TP5, 21 \& 22) (Figs. 2-5). The test pit soil profiles were described (Table 2) and 84 bulk soil samples were collected for physical and chemical analyses, and seven soil blocks taken for soil micromorphological analysis.

The test pits revealed a quite consistent set of profiles through both terrace systems. The soil profiles comprised either just thin ploughsoils over the weathered tonalite bedrock, or the ploughsoil over a depleted zone of terrace made-ground which buried a remnant of a probable former thin cambisol soil profile developed on the weathered tonalite bedrock. Small bulk and micromorphological samples were collected from one $1 \times 1 \mathrm{~m}$ test pit and of two $50 \times 50 \mathrm{~cm}$ test pits at six levels from the main representative soil horizons across the terrace system. The test pits were dug to the base of the buried B horizon and/or top of the weathered tonalite parent material (Figs. 4 \& 5).

Samples were subjected to light grinding using a pestle and mortar before being sieved through a $2 \mathrm{~mm}$ mesh sieve, then processed using

Table 1

Summary of methods used to discern agricultural inputs ( $\mathrm{OM}=$ organic matter; Na-Hex $=$ sodium hexametaphosphate).

\begin{tabular}{|c|c|c|}
\hline Agricultural input & Publication & Methods used \\
\hline Tethering to an argillic horizon & $\begin{array}{l}\text { Eash and Sandor (1995); Goodman-Elgar (2002, 2008); Homburg } \\
\text { et al. (2005); Norton et al. (2003); Sandor and Eash }(1991,1995)\end{array}$ & $\begin{array}{l}\text { Particle size analysis (pipette and hydrometer methods following } \\
\text { removal of OM and salts, dispersed with Na-Hex) and soil } \\
\text { micromorphology }\end{array}$ \\
\hline Fertiliser application & $\begin{array}{l}\text { Brooks (1998); Goodman-Elgar (2002), (2008); Hastorf (1993); } \\
\text { Homburg et al. (2005); Sandor and Eash (1991, 1995) }\end{array}$ & $\begin{array}{l}\text { Bulk density; pH; organic C; total N; total and available P } \\
\text { Olsen and Sommers (1982) }\end{array}$ \\
\hline Corralling on fields & Goodman-Elgar (2002); Hastorf (1993); Lane (2006) & As above, but only distinguishable from each other through historical \\
\hline $\begin{array}{l}\text { Application of manure from corrals } \\
\text { away from field }\end{array}$ & Goodman-Elgar (2002); Hastorf (1993); Lane (2006) & $\begin{array}{l}\text { documentation, ethnographic observation \&/or soil } \\
\text { micromorphology }\end{array}$ \\
\hline Middening & $\begin{array}{l}\text { Brooks (1998); Goodman-Elgar (2002, 2008); Sandor and Eash } \\
(1991,1995)\end{array}$ & $\begin{array}{l}\text { The presence of artefacts such as ceramics throughout the epipedon } \\
\text { during excavation and the geo-chemical methods mentioned above }\end{array}$ \\
\hline Burning & Goodman-Elgar (2002); Hastorf (1993); Sandor and Eash (1995) & $\begin{array}{l}\text { Profile excavation and observation soil micromorphology, and total } \\
\text { and available P } \\
\text { Olsen and Sommers (1982) }\end{array}$ \\
\hline
\end{tabular}




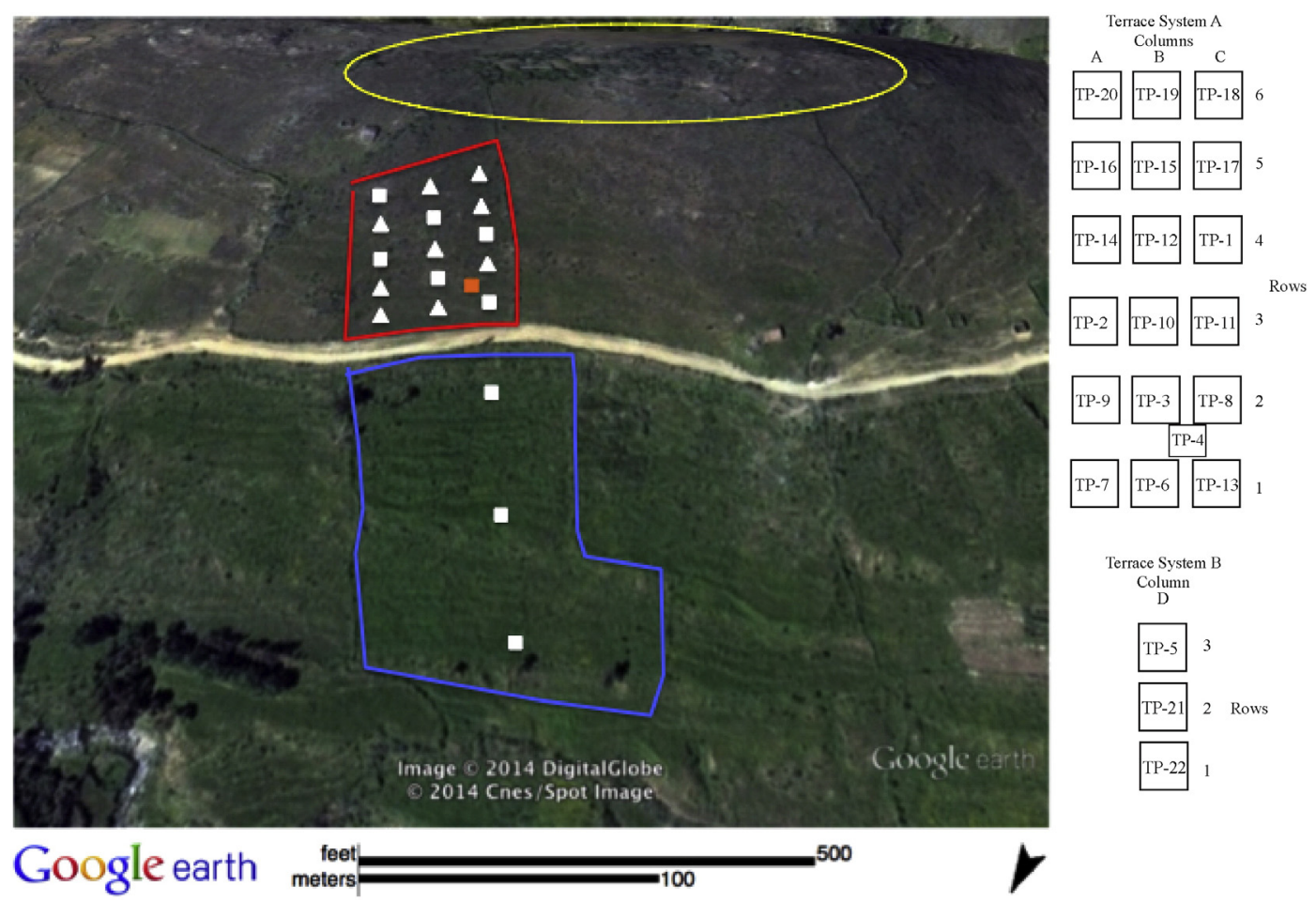

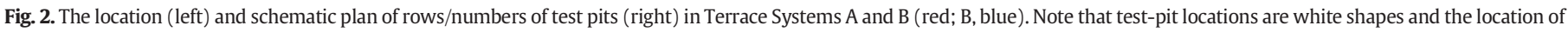
Viejo Sangayaico site is circled in yellow. (W. Nanavati). (For interpretation of the references to colour in this figure legend, the reader is referred to the web version of this article.) Image of location based on Google Earth: Image@ 2014 Digital Globe/@2014 Cnes/Spot Image.

the suite of analyses described in Table 3 (with references therein; Soil Survey Staff, 1993), which includes, pH, electrical conductivity (EC), organic matter content (loss-on-ignition), soil moisture content, particle size analysis, phosphorus (P) content and soil micromorphology. Given the tonalitic parent material and various sand-sized aggregates present in the soil samples, some methodological adaptations were necessary to produce useful data. For the organic matter content and particle size analyses, sodium hexametaphosphate was used as a deflocculant to achieve a $\mathrm{pH}$ of 9 and disperse the sample fabric and break-down the clay (kaolinite) component (Devesa-Rey et al., 2011; Dwomo and Dedzoe, 2010; El-Swaify, 1980; Gee and Bauder, 1986; Goodman-Elgar, 2008; Silva et al., 2015), as well as a vortex and rotor mixer and sub-sampling from the mid-point of the suspension for particle size analysis using the Malvern Mastersizer S Laser Diffraction Analyser (Gee and Bauder, 1986). To account for the inclusion of medium-grained sand in the samples processed by laser diffraction,

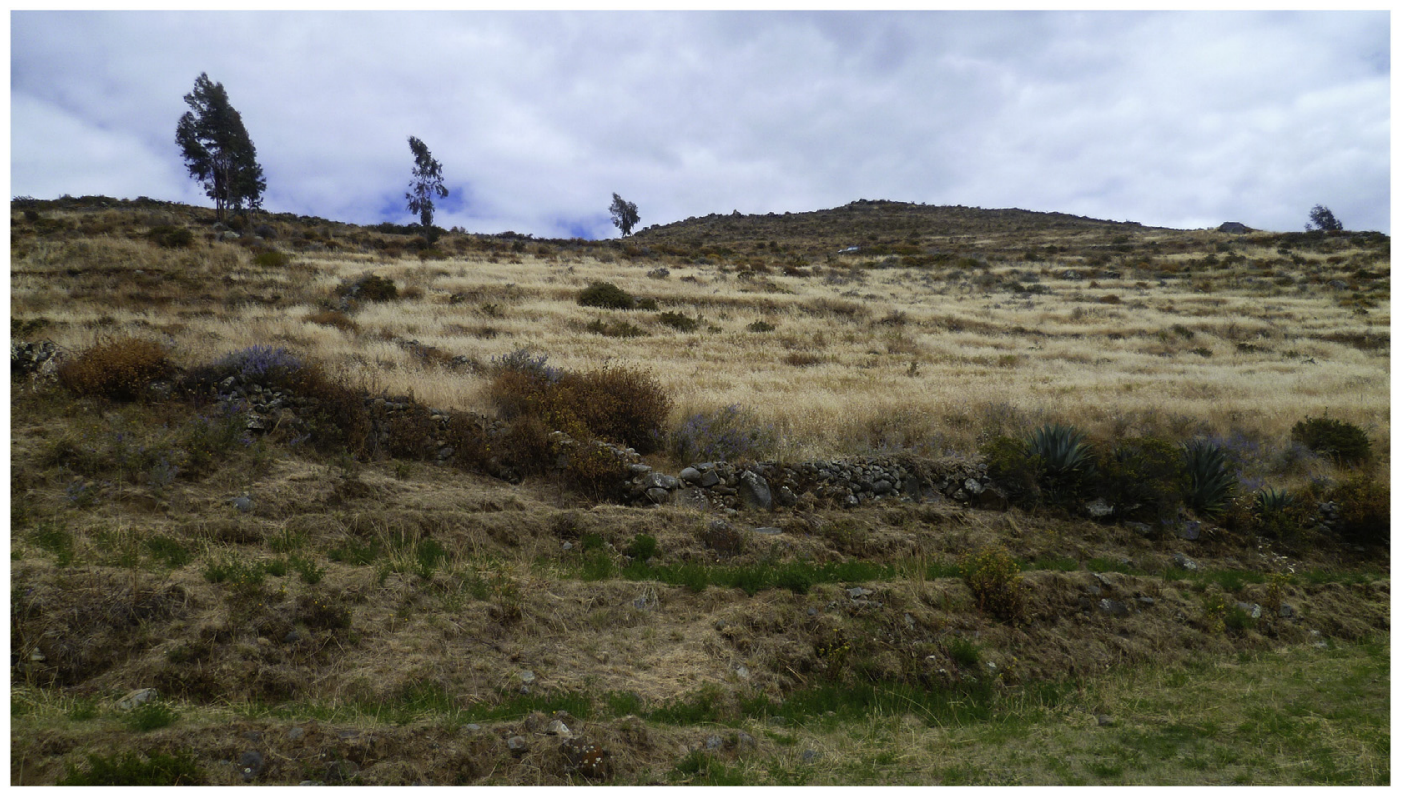

Fig. 3. A view of terrace system B today looking up-slope to the Viejo Sangayaico sites. C. French. 


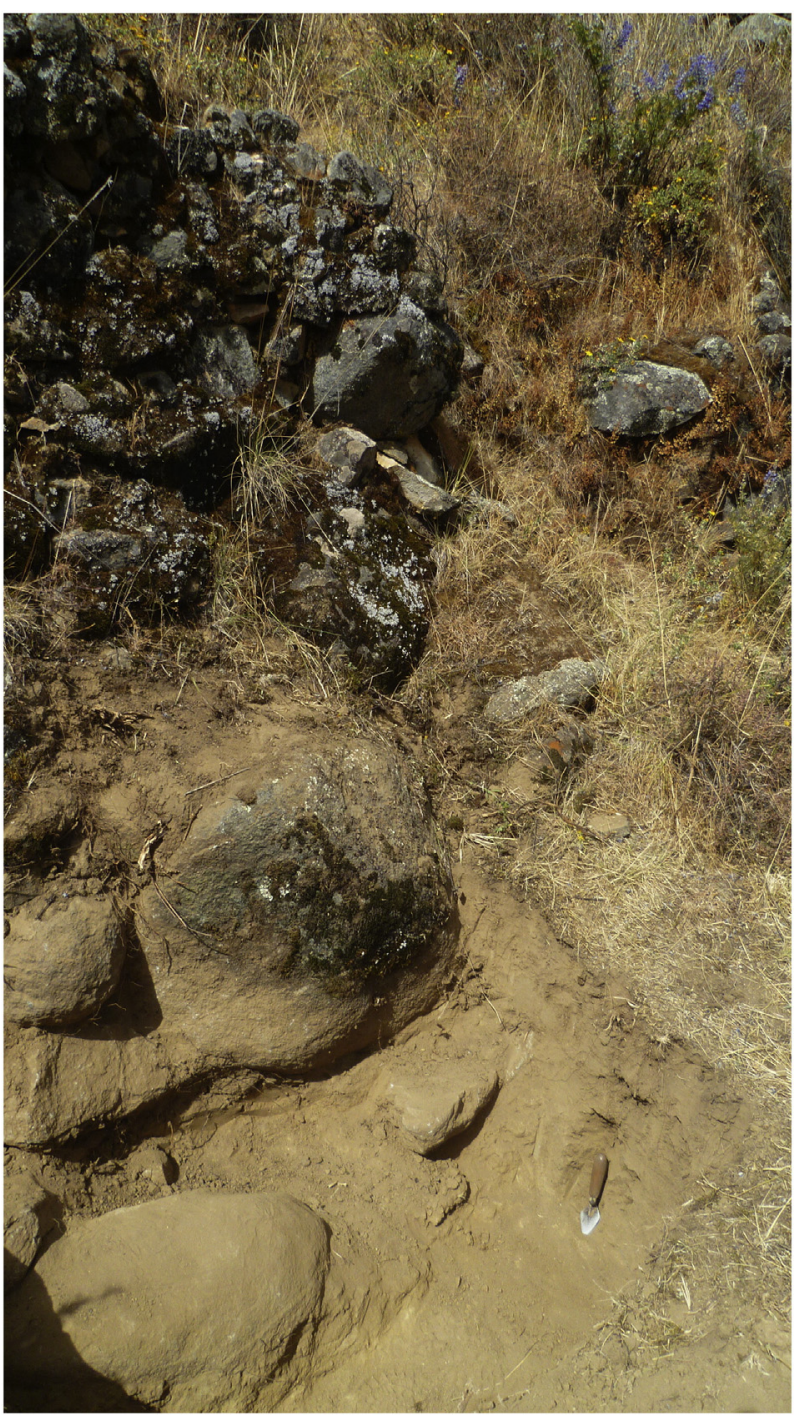

Fig. 4. Terrace wall and profile photograph of Test Pit 1 in terrace system A. C. French.

medium-sand values from wet-sieving and laser diffraction were combined to decrease distribution errors.

Unfortunately without the use of radiocarbon or optically stimulated luminescence (OSL) dating, the excavated terrace systems can only be relatively dated by assuming relationships to the occupation of the associated Sangayaico site. There was no evident organic material from the soil profiles in the test pits that was suitable for radiometric dating, and there were insufficiently clear contacts between the terrace make-up deposits and possible buried soils to justify sampling for OSL dating. Consequently the field systems only have a relative chronology through the terrace systems association with the settlement sites on the Sangayaico ridge above. There is now a series of radiocarbon dates ranging from cal AD 1100-1500 (OxA-30914/15/16, OxA-30930/31) for the Late Intermediate site from the associated excavations at Sangayaico (Lane et al., 2015). Another limitation met in the field was the fact that the entirety of the arable landscape has been cultivated, leaving no natural controls to test against.

Finally, a series of seven blocks were taken from Test Pits 1,2, 4 and 5 for soil micromorphological analysis (Courty et al., 1989; Bullock et al., 1985; Murphy, 1986; Stoops, 2003; Stoops et al., 2010). These aimed to be representative of, and characterise, the main stratigraphic horizons present in the terrace system. Their analysis would serve to ground-truth the other physical analyses, and indicate the pedogenic processes at work. This was part of a wider geoarchaeological study of the upper Ica valley (French, 2015, pp. 54-62).

\section{Results}

The results of our study are summarised in Tables 2-7. The research questions considered are addressed at the scale of the individual profile, transect, and terrace system. The quantitative results demonstrate some down-profile and downslope trends (Table 6), and in combination with the micromorphological analyses (Table 7), the assembled data create a clear picture of the inherent soil characteristics and processes of the Sangayaico terrace soils.

\subsection{The test pit profiles}

For the majority of test pit profiles four soil horizons were evident (Table 2; Fig. 5). These comprised a modern plough zone (or Ap) and an eluvial (or Eb) horizon, both fine sandy loams which have developed in the upcast soil of variable thicknesses used to construct the terrace, overlying a variable thickness $(c .8-25 \mathrm{~cm})$ of a buried, fine sandy/silty clay loam soil which is probably a former A horizon (labelled bA2), all developed on the weathered tonalite bedrock. In seven of the test pits (TP 3, 8, 9, 16, 18, 21 and 22), including most of Terrace System B downslope (TP 21 and 22), there was no evidence of a buried soil or former A horizon surviving at the base of the profile, with the terrace deposits situated directly on the bedrock. The test pits in the lower Terrace System B also exhibited the greatest profile depths of $c .56-67 \mathrm{~cm}$.

\section{2. $\mathrm{pH}$ and electrical conductivity}

Soil pH ranged from weakly acidic to weakly calcareous with a range of values from 5.33 to 7.19 and an average of 6.34 (Tables 4-6). Terrace System A was more neutral in $\mathrm{pH}$ range; Terrace System B was more weakly calcareous to neutral in range. Lower values of $\mathrm{pH}$ and concomitant low concentrations of inorganic carbon are common among soil systems on volcanic substrates such a tonalite (Nettleton et al., 1970). Down-profile, $\mathrm{pH}$ values varied little and mainly remained in the neutral range, whereas down-slope the trend is for the profiles to become slightly more calcareous.

Electrical conductivity ranged from 26.6 to $178.8 \mu \mathrm{S} / \mathrm{m}$, averaging $57.1 \mu \mathrm{S} / \mathrm{m}$ (Tables 4 and 5). These values are all relatively low and do not suggest a high potential for elemental changes and reactions taking place in this soil system. The highest EC values were from the uppermost growing horizon, a common feature in arid environments due to the deposition of salts in the topsoil during evapo-transpiration and the breakdown of organic matter (Meurisse et al., 1990; Rhoades et al., 1999; Smith et al., 1996).

\subsection{Soil moisture and organic contents}

The soil moisture content of the air-dried soil ranged from 0.86 to $6.05 \%$, averaging $2.39 \%$, but increased down-profile by as much as $4 \%$ (Tables 4-6). In Terrace System A, the soil moisture content for the upper half of the profile was quite low but even, with the lower parts of the profiles showing greater variability, fluctuating between 1.41 to $4.48 \%$ and 1.65 to $6.05 \%$.

The soil organic matter data from the loss-on-ignition analysis (LOI 500 and Leco TruSpec) ranged from 2.1 to $7.17 \%$, with two outlier high values of $8.8 \%$ in the A horizons of TP 1 and $10.89 \%$ in TP18 (Tables 4-6). In Terrace System A, the soil organic matter content generally remained quite stable to slightly decreasing by $1-4 \%$ down-profile, with slight enhancement in the A horizon. In Terrace System B, the organic matter content remained quite steady with a range of 4.79$5.73 \%$, except for $8-10.54 \%$ high values in the $\mathrm{Ah} / \mathrm{Eb}$ horizons of TP5. 
Upper, Middle, and Lower Terrace Diagrams from Terrace System A

Down Slope

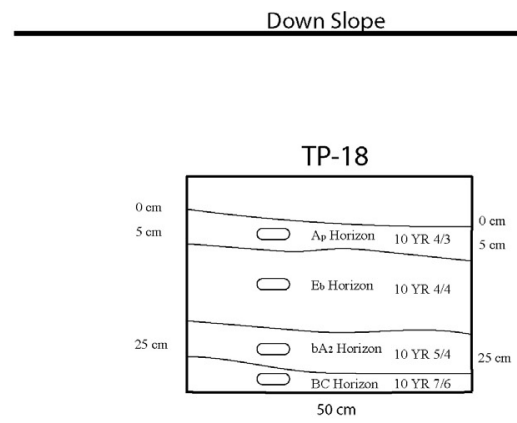

TP-1

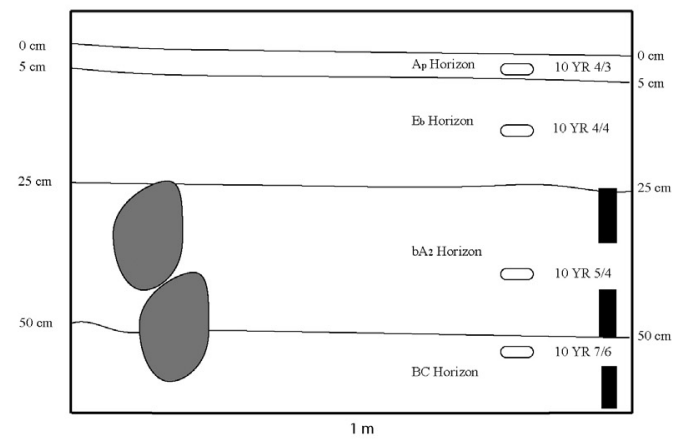

TP-13

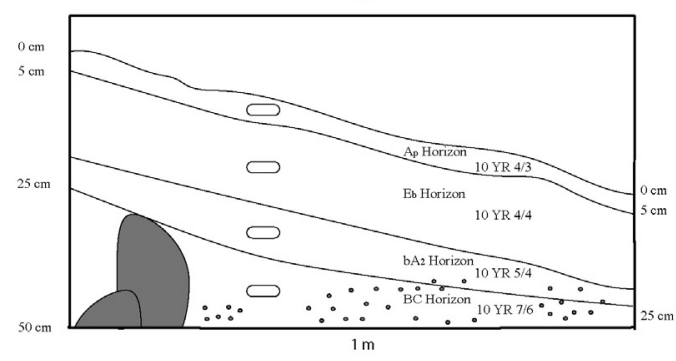

Terrace Diagrams from Terrace System B
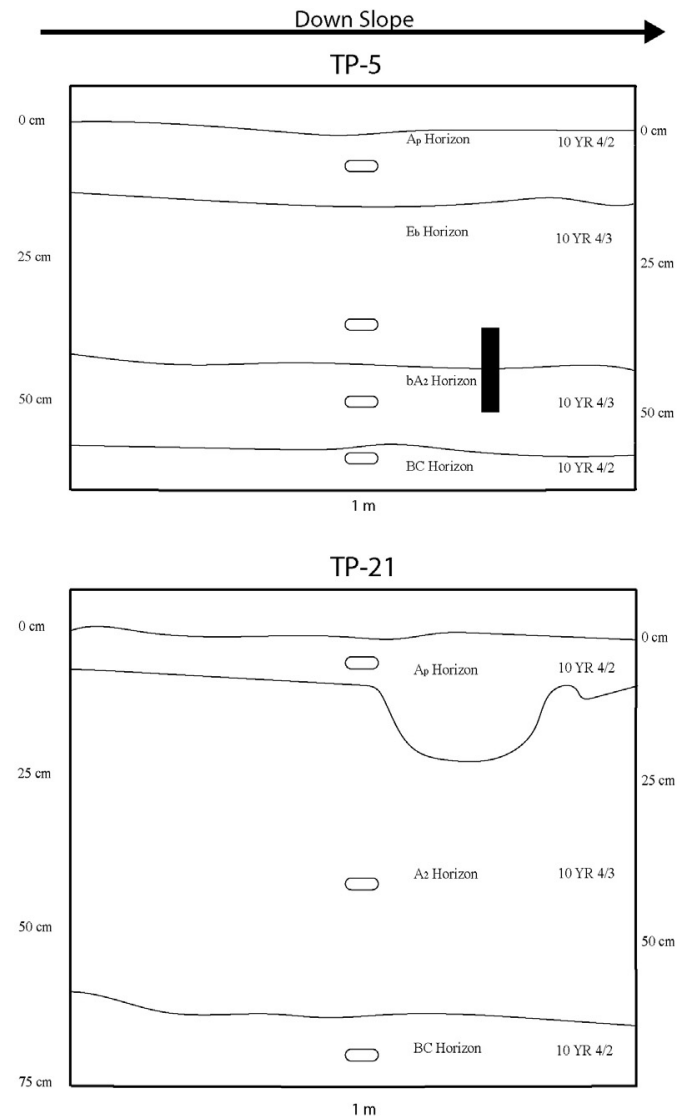

TP-22

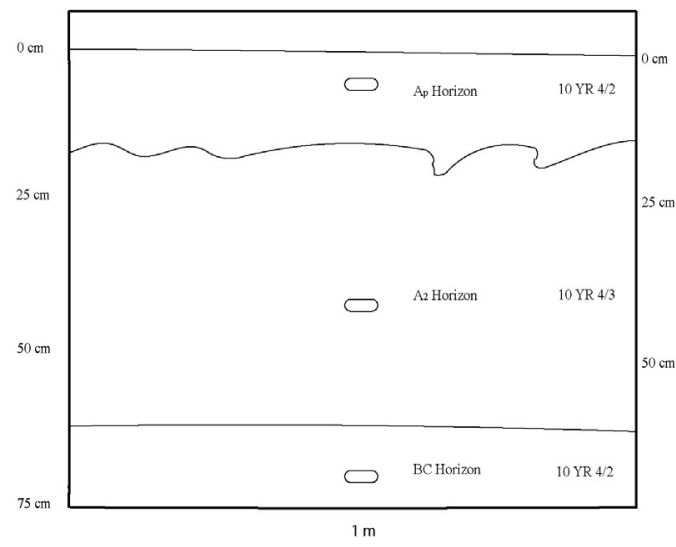

Fig. 5. Selected sections of TP1, 13 and 18 in Terrace System A, and TP5, 21 and 22 in Terrace System B at Sangayaico. W. Nanavati.

\subsection{Particle size analysis}

Due to the variability of the sand- and silt-sized particle distribution, no clear patterns were evident down-profile in either terrace system. However, the percentage distribution of the clay-sized fraction generally decreased down-profile, with variability increasing downslope (only in Terrace System A). In Terrace System A Row 1, there was a downprofile increase in the distribution of clay, most notably between layers 3 and 4 or in the buried B soil, and between Rows 3 and 1 the clay component increased to $11 \%$ with a coincident increase in silt content. Terrace System B showed little soil textural variation downslope, with the middle terrace (Row 2) showing a higher distribution of sand at the expense of the silt- and clay-sized fractions.

\subsection{Phosphorous determination}

Phosphorous determination results were separated in this study into total $\left(\mathrm{P}_{\text {tot }}\right)$, inorganic $\left(\mathrm{P}_{\text {in }}\right)$ and organic $\left(\mathrm{P}_{\text {org }}\right)$ categories. Phosphorus values averaged $788.17 \mu \mathrm{g} / \mathrm{ml}_{\text {tot }}, 569.29 \mu \mathrm{g} / \mathrm{mL} \mathrm{P}_{\mathrm{in}}$, and $218.88 \mu \mathrm{g} / \mathrm{ml}$ $\mathrm{P}_{\text {org }}$ (Tables 4 and 5). The results showed no general trends downprofile (Table 6 ), but there was a large range in $P$ values represented from weakly to moderately enhanced $\left(P_{\text {tot }}=224.91-1300.76 \mu \mathrm{g} / \mathrm{ml}\right.$ 
Table 2

Field description of the test pits at Sangayaico (note: Profiles 1-4 and 6-20 were taken from Terrace System A; Profiles 5, 21 and 22 were taken from Terrace System B).

\begin{tabular}{|c|c|c|}
\hline $\begin{array}{l}\text { Test pit: UTM } \\
\text { location \& } \\
\text { elevation }\end{array}$ & $\begin{array}{l}\text { Depth below } \\
\text { ground surface } \\
(\mathrm{cm})\end{array}$ & Description \\
\hline TP1: & $0-5$ & Loam; 10YR4/3; friable; granular; roots present, $<2 \mathrm{~mm}$; ploughsoil or Ap \\
\hline $\begin{array}{l}\text { 18L } 0475586 \\
8474994\end{array}$ & $5-25$ & $\begin{array}{l}\text { Sandy loam; } 10 \text { YR4/4; friable; blocky; roots present to about } 8 \mathrm{~cm},<2 \mathrm{~mm} ;<3 \% \text { charcoal, } 1-2 \mathrm{~cm} \text {; depleted terrace } \\
\text { make-up soil or Eb }\end{array}$ \\
\hline \multirow[t]{2}{*}{3553 m.a.s.l. } & $25-50$ & $\begin{array}{l}\text { Silty clay loam; } 10 \mathrm{YR} 5 / 4 \text {; firm; massive; rare roots, }<3 \% \text { charcoal, } 1-2 \mathrm{~cm} \text {; mottling } 1-2 \mathrm{~cm}, 10 \mathrm{YR} 8 / 2 \text {; probable buried } \\
\text { former soil or bA2-bB profile }\end{array}$ \\
\hline & $50+$ & Silt loam with abundant fine tonalite fragments; 10YR7/6; extra firm; massive; very weathered parent material; B/C \\
\hline TP2: & $0-10$ & Loam; 10YR4/3; friable; granular; roots present to about $8 \mathrm{~cm},<2 \mathrm{~mm}$; Ap \\
\hline 18L 04756138475024 & $10-21$ & Sandy loam; 10YR4/4; friable; blocky; <3\% charcoal, $1-2 \mathrm{~cm}$; Eb \\
\hline \multirow[t]{2}{*}{3612 m.a.s.l. } & $21-40$ & $\begin{array}{l}\text { Silty clay loam; } 10 \text { YR } 5 / 4 \text {; firm; massive; rare roots, }<3 \% \text { charcoal, } 1-2 \mathrm{~cm} \text {; mottling } 1-2 \mathrm{~cm}, 10 \mathrm{YR} 8 / 2 \text {; probable former } \\
\text { buried soil or bA2-bB profile }\end{array}$ \\
\hline & $40+$ & Silt loam with abundant fine tonalite fragments; 10YR7/6; extra firm; massive; very weathered parent material; B/C \\
\hline TP3: & $0-15$ & Loam; 10YR5/3; friable; granular; roots present to about $8 \mathrm{~cm},<2 \mathrm{~mm}$; Ap \\
\hline $\begin{array}{l}\text { 18L } 04755928475024 \\
3606 \text { m.a.s.l. }\end{array}$ & $15+$ & Silt loam with abundant fine tonalite fragments; 10YR5/6; extra firm; massive; very weathered parent material; B/C \\
\hline TP4: $\quad$ : & $0-5$ & Loam; 10YR5/44; friable; granular; roots present to about $8 \mathrm{~cm},<2 \mathrm{~mm}$; Ap \\
\hline 18L 04755718475024 & $5-22$ & Sandy loam; 10YR5/6; friable; blocky; roots present; $<3 \%$ charcoal, $1-2 \mathrm{~cm}$; Eb \\
\hline \multirow[t]{2}{*}{3604 m.a.s.l. } & $22-46$ & $\begin{array}{l}\text { Silty clay loam with increasing fine tonalite fragments down-profile; } 10 \text { YR5 } / 4 \text {; firm; massive; rare roots, }<3 \% \text { charcoal, } \\
1-2 \mathrm{~cm} \text {; mottling } 1-2 \mathrm{~cm}, 10 \text { YR } 8 / 2 \text {; probable former buried soil or bA2-bB profile }\end{array}$ \\
\hline & $46+$ & Silt loam with abundant fine tonalite fragments; 10YR7/6; extra firm; massive; very weathered parent material; B/C \\
\hline \multirow{4}{*}{$\begin{array}{l}\text { TP5: } \\
\text { 18L } 04755398475068 \\
\text { 3586 m.a.s.l. }\end{array}$} & $0-14$ & Loam; 10YR4/2; friable; granular; roots present to about $8 \mathrm{~cm},<2 \mathrm{~mm}$; Ap \\
\hline & $14-44$ & $\begin{array}{l}\text { Sandy loam; 10YR4/3; friable; blocky; roots present to about } 20 \mathrm{~cm},<2 \mathrm{~mm} ; 5-10 \% \text { charcoal, } 1-2 \mathrm{~cm} \text {; few undiagnostic } \\
\text { ceramic fragments; Eb }\end{array}$ \\
\hline & 44-56 & $\begin{array}{l}\text { Sandy loam; 10YR4/3; friable; blocky; roots present, }<2 \mathrm{~mm} ; 5-10 \% \text { charcoal, } 1-2 \mathrm{~cm} \text {; probable former buried soil or } \\
\text { bA2-bB profile }\end{array}$ \\
\hline & $56+$ & Silt loam with abundant fine tonalite fragments; 10YR4/2; extra firm; massive; very weathered parent material; B/C \\
\hline \multirow{4}{*}{$\begin{array}{l}\text { TP6: } \\
\text { 18L } 04755858475046 \\
3609 \text { m.a.s.l. }\end{array}$} & $0-5$ & Loam; 10YR4/3; friable; granular; roots present, $<2$ mm; Ap \\
\hline & $5-17$ & Sandy loam; 10YR5/4; friable; blocky; roots present to about $8 \mathrm{~cm},<2 \mathrm{~mm} ;<3 \%$ charcoal, $1-2 \mathrm{~cm}$; Eb \\
\hline & $17-24$ & $\begin{array}{l}\text { Silty clay loam; 10YR5/4; firm; massive; rare roots, }<3 \% \text { charcoal, } 1-2 \mathrm{~cm} \text {; mottling } 1-2 \mathrm{~cm}, 10 \mathrm{YR} 8 / 2 \text {; probable buried } \\
\text { bA2 }\end{array}$ \\
\hline & $24-46$ & Silt loam with abundant fine tonalite fragments; 10YR7/6; extra firm; massive; very weathered parent material; B/C \\
\hline \multirow{4}{*}{$\begin{array}{l}\text { TP7: } \\
\text { 18L } 04755948475050 \\
3609 \text { m.a.s.l. }\end{array}$} & $0-3$ & Loam; 10YR4/3; friable; granular; roots present, <2 mm; Ap \\
\hline & $3-7$ & Sandy loam; 10YR4/4; friable; blocky; roots present, $<2 \mathrm{~mm} ;<3 \%$ charcoal, $1-2 \mathrm{~cm}$; Eb \\
\hline & $7-24$ & $\begin{array}{l}\text { Silty clay loam; } 10 \text { YR5/4; firm; massive; rare roots, }<3 \% \text { charcoal, } 1-2 \mathrm{~cm} \text {; mottling } 1-2 \mathrm{~cm}, 10 \mathrm{YR} 8 / 2 \text {; probable former } \\
\text { buried soil or bA2 }\end{array}$ \\
\hline & $24-46$ & Silt loam with abundant fine tonalite fragments; 10YR7/6; extra firm; massive; very weathered parent material; B/C \\
\hline \multirow{3}{*}{$\begin{array}{l}\text { TP8: } \\
\text { 18L } 04755718475014 \\
3613 \text { m.a.s.l. }\end{array}$} & $0-3$ & Loam; 10YR5/4; friable; granular; roots present, $<2$ mm; Ap \\
\hline & $3-23$ & Sandy loam; 10YR5/6; friable; blocky; roots present to about $8 \mathrm{~cm},<2 \mathrm{~mm}$; Eb \\
\hline & $23+$ & Silt loam; 10YR7/6; extra firm; massive; very weathered parent material; B/C \\
\hline \multirow{7}{*}{$\begin{array}{l}\text { TP9: } \\
\text { 18L } 04756058475038 \\
\text { 3615 m.a.s.l. } \\
\text { TP10: } \\
\text { 18L } 04755948475006 \\
\text { 3620 m.a.s.l. }\end{array}$} & $0-2$ & Loam; 10YR5/4; friable; granular; roots present, $<2$ mm; Ap \\
\hline & $2-10$ & Sandy loam; 10YR5/6; friable; blocky; roots present to about $8 \mathrm{~cm},<2 \mathrm{~mm}$; Eb \\
\hline & $10+$ & Silt loam with abundant fine tonalite fragments; 10YR7/6; extra firm; massive; very weathered parent material; B/C \\
\hline & $0-5$ & Loam; 10YR4/3; friable; granular; roots present to about $8 \mathrm{~cm},<2 \mathrm{~mm}$; Ap \\
\hline & $5-24$ & Sandy loam; 10YR4/4; friable; blocky; roots present to about $8 \mathrm{~cm},<2 \mathrm{~mm} ;<3 \%$ charcoal $1-2 \mathrm{~cm}$; Eb \\
\hline & 24-43 & $\begin{array}{l}\text { Silty clay loam; } 10 \text { YR5/4; firm; massive; rare roots, }<3 \% \text { charcoal, } 1-2 \mathrm{~cm} \text {; mottling } 1-2 \mathrm{~cm}, 10 \text { YR } 8 / 2 \text {; probable former } \\
\text { buried soil or bA2-bB profile }\end{array}$ \\
\hline & $43+$ & Silt loam with abundant fine tonalite fragments; 10YR7/6; extra firm; massive; very weathered parent material; B/C \\
\hline \multirow{4}{*}{$\begin{array}{l}\text { TP11: } \\
\text { 18L } 04755778475002 \\
\text { 3620 m.a.s.l. }\end{array}$} & $0-5$ & Loam; 10YR4/3; friable; granular; roots present, $<2$ mm; Ap \\
\hline & $5-16$ & Sandy loam; 10YR4/4; friable; blocky; roots present to about $8 \mathrm{~cm},<2 \mathrm{~mm} ;<3 \%$ charcoal, $1-2 \mathrm{~cm}$; Eb \\
\hline & $16-36$ & $\begin{array}{l}\text { Silty clay loam; 10YR5/4; firm; massive; rare roots, }<3 \% \text { charcoal, } 1-2 \mathrm{~cm} \text {; mottling } 1-2 \mathrm{~cm}, 10 \text { YR } 8 / 2 \text {; probable former } \\
\text { buried soil or bA2-bB profile }\end{array}$ \\
\hline & $36+$ & Silt loam with abundant fine tonalite fragments; 10YR7/6; extra firm; massive; very weathered parent material; B/C \\
\hline \multirow{4}{*}{$\begin{array}{l}\text { TP12: } \\
\text { 18L } 04756038475008 \text {; } \\
\text { 3623 m.a.s.l. }\end{array}$} & $0-4$ & Loam; 10YR4/3; friable; granular; roots present, <2 mm; Ap \\
\hline & $4-18$ & Sandy loam; 10YR4/4; friable; blocky; roots present to about $8 \mathrm{~cm},<2 \mathrm{~mm} ;<3 \%$ charcoal, $1-2 \mathrm{~cm}$; Eb \\
\hline & $18-37$ & $\begin{array}{l}\text { Silty clay loam; } 10 \text { YR } 5 / 4 \text {; firm; massive; rare roots, }<3 \% \text { charcoal, } 1-2 \mathrm{~cm} \text {; mottling } 1-2 \mathrm{~cm}, 10 \mathrm{YR} 8 / 2 \text {; probable former } \\
\text { buried soil or bA2-bB profile }\end{array}$ \\
\hline & $37+$ & Silt loam with abundant fine tonalite fragments; 10YR7/6; extra firm; massive; very weathered parent material; B/C \\
\hline \multirow{4}{*}{$\begin{array}{l}\text { TP13: } \\
\text { 18L } 04755638475026 \\
\text { 3610 m.a.s.l. }\end{array}$} & $0-5$ & Loam; 10YR4/3; friable; granular; roots present, $<2$ mm; Ap \\
\hline & $5-17$ & Sandy loam; 10YR4/4; friable; blocky; roots present to about $8 \mathrm{~cm},<2 \mathrm{~mm}$; $<3 \%$ charcoal, $1-2 \mathrm{~cm}$; Eb \\
\hline & $17-24$ & $\begin{array}{l}\text { Silty clay loam; } 10 \text { YR } 5 / 4 \text {; firm; massive; rare roots, }<3 \% \text { charcoal } 1-2 \mathrm{~cm} \text {; mottling } 1-2 \mathrm{~cm}, 10 \text { YR } 8 / 2 \text {; probable former } \\
\text { buried soil or bA2-bB profile }\end{array}$ \\
\hline & $24-46$ & Silt loam with abundant fine tonalite fragments; 10YR7/6; extra firm; massive; very weathered parent material; B/C \\
\hline \multirow{4}{*}{$\begin{array}{l}\text { TP14: } \\
\text { 18L } 04756168475018 \\
\text { 3623 m.a.s.l. }\end{array}$} & $0-6$ & Loam; 10YR4/3; friable; granular; roots present, $<2$ mm; Ap \\
\hline & $6-20$ & Sandy loam; 10YR4/4; friable; blocky; roots present to about $8 \mathrm{~cm},<2 \mathrm{~mm} ;<3 \%$ charcoal, $1-2 \mathrm{~cm}$; Eb \\
\hline & $20-30$ & $\begin{array}{l}\text { Silty clay loam; } 10 \mathrm{YR} 5 / 4 \text {; firm; massive; rare roots, }<3 \% \text { charcoal, } 1-2 \mathrm{~cm} \text {; mottling } 1-2 \mathrm{~cm}, 10 \mathrm{YR} 8 / 2 \text {; probable former } \\
\text { buried soil or bA2-bB profile }\end{array}$ \\
\hline & $30+$ & Silt loam with abundant fine tonalite fragments; 10YR7/6; extra firm; massive; very weathered parent material; B/C \\
\hline \multirow{4}{*}{$\begin{array}{l}\text { TP15: } \\
\text { 18L } 04756148474994 \\
\text { 3629 m.a.s.l. }\end{array}$} & $0-5$ & Loam; 10YR4/3; friable; granular; roots present, <2 mm; Ap \\
\hline & $5-28$ & Sandy loam; 10YR4/4; friable; blocky; roots present to about $8 \mathrm{~cm},<2 \mathrm{~mm} ;<3 \%$ charcoal, $1-2 \mathrm{~cm}$; Eb \\
\hline & $28-58$ & $\begin{array}{l}\text { Silty clay loam; } 10 \text { YR } 5 / 4 \text {; firm; massive; rare roots, }<3 \% \text { charcoal, } 1-2 \mathrm{~cm} \text {; mottling } 1-2 \mathrm{~cm}, 10 \mathrm{YR} 8 / 2 \text {; probable former } \\
\text { buried soil or bA2-bB profile }\end{array}$ \\
\hline & $\begin{array}{l}58+ \\
0-5\end{array}$ & $\begin{array}{l}\text { Silt loam with abundant fine tonalite fragments; 10YR7/6; extra firm; massive; very weathered parent material; B/C } \\
\text { Loam; 10YR4/3; friable; granular; roots present, }<2 \mathrm{~mm} \text {; Ap }\end{array}$ \\
\hline
\end{tabular}


Table 2 (continued)

\begin{tabular}{|c|c|c|}
\hline $\begin{array}{l}\text { Test pit: UTM } \\
\text { location \& } \\
\text { elevation }\end{array}$ & $\begin{array}{l}\text { Depth below } \\
\text { ground surface } \\
(\mathrm{cm})\end{array}$ & Description \\
\hline 18L 04756248475006 & $5-31$ & Sandy loam; 10YR4/4; friable; blocky; roots present to about $8 \mathrm{~cm},<2 \mathrm{~mm} ;<3 \%$ charcoal, $1-2 \mathrm{~cm}$; Eb \\
\hline \multirow[t]{3}{*}{3628 m.a.s.l. } & $31-50$ & $\begin{array}{l}\text { Silty clay loam; } 10 \text { YR5 } / 4 \text {; firm; massive; rare roots, }<3 \% \text { charcoal, } 1-2 \mathrm{~cm} \text {; mottling } 1-2 \mathrm{~cm}, 10 \text { YR } 8 / 2 \text {; probable former } \\
\text { buried soil or bA2 }\end{array}$ \\
\hline & $50-57$ & Sandy loam; 5G8/1 \& N1; friable; granular; ? Eb2 \\
\hline & $57+$ & Silt loam with abundant fine tonalite fragments; 10YR7/6; extra firm; massive; very weathered parent material; B/C \\
\hline TP17: & $0-5$ & Loam; 10YR4/3; friable; granular; roots present to about $8 \mathrm{~cm},<2 \mathrm{~mm}$; Ap \\
\hline 18L 04755988474980 & $5-20$ & Sandy loam; 10YR4/4; friable; blocky; roots present to about $8 \mathrm{~cm},<2 \mathrm{~mm} ;<3 \%$ charcoal, $1-2 \mathrm{~cm}$; Eb \\
\hline \multirow[t]{2}{*}{3628 m.a.s.l. } & 20-39 & $\begin{array}{l}\text { Silty clay loam; } 10 \text { YR } 5 / 4 \text {; firm; massive; rare roots, }<3 \% \text { charcoal, } 1-2 \mathrm{~cm} \text {; mottling } 1-2 \mathrm{~cm}, 10 \text { YR } 8 / 2 \text {; probable former } \\
\text { buried soil or bA2-bB profile }\end{array}$ \\
\hline & $39+$ & Silt loam with abundant fine tonalite fragments; 10YR7/6; extra firm; massive; very weathered parent material; B/C \\
\hline TP18: & $0-8$ & Loam; 10YR4/3; friable; granular; roots present, $<2$ mm; Ap \\
\hline $\begin{array}{l}\text { 18L } 04756138474972 \\
3634 \text { m.a.s.l. }\end{array}$ & $8-20$ & Sandy loam; 10YR4/4; friable; blocky; roots present to about $8 \mathrm{~cm},<2 \mathrm{~mm} ;<3 \%$ charcoal, $1-2 \mathrm{~cm}$; Eb \\
\hline TP19: & $0-10$ & Loam; 10YR4/3; friable; granular; roots present to about $8 \mathrm{~cm},<2 \mathrm{~mm}$; Ap \\
\hline 18L 04756258474982 & $10-22$ & Sandy loam; 10 YR4/4; friable; blocky; $<3 \%$ charcoal, $1-2 \mathrm{~cm}$; Eb \\
\hline \multirow[t]{2}{*}{3636 m.a.s.l. } & $22-33$ & $\begin{array}{l}\text { Silty clay loam; } 10 \text { YR } 5 / 4 \text {; firm; massive; rare roots, }<3 \% \text { charcoal, } 1-2 \mathrm{~cm} \text {; mottling } 1-2 \mathrm{~cm}, 10 \text { YR } 8 / 2 \text {; probable former } \\
\text { buried soil or bA2-bB profile }\end{array}$ \\
\hline & $33+$ & Silt loam with abundant fine tonalite fragments; 10YR7/6; extra firm; massive; very weathered parent material; B/C \\
\hline TP20: & $0-6$ & Loam; 10YR4/3; friable; granular; roots present, $<2$ mm; Ap \\
\hline 18L 04756348474998 & $6-20$ & Sandy loam; 10YR4/4; friable; blocky; roots present to about $8 \mathrm{~cm},<2 \mathrm{~mm} ;<3 \%$ charcoal, $1-2 \mathrm{~cm}$; Eb \\
\hline \multirow[t]{2}{*}{3633 m.a.s.l. } & $20-47$ & $\begin{array}{l}\text { Silty clay loam; } 10 \text { YR } 5 / 4 \text {; firm; massive; rare roots, }<3 \% \text { charcoal, } 1-2 \mathrm{~cm} \text {; mottling } 1-2 \mathrm{~cm}, 10 \text { YR } 8 / 2 \text {; probable former } \\
\text { buried soil or bA2-bB profile }\end{array}$ \\
\hline & $47+$ & Silt loam with abundant fine tonalite fragments; 10YR7/6; extra firm; massive; very weathered parent material; B/C \\
\hline TP21: & $0-7$ & Loam; 10YR4/2; friable; granular; roots present, $<2 \mathrm{~mm}$; Ap \\
\hline 18L 04755108475102 & $7-67$ & Sandy loam; 10YR4/3; friable; blocky; roots present to about $20 \mathrm{~cm}<2 \mathrm{~mm} ; 5-10 \%$ charcoal, $1-2 \mathrm{~cm}$; A2 \\
\hline 3574 m.a.s.l. & $67+$ & Silt loam with abundant fine tonalite fragments; 10YR4/2; extra firm; massive; very weathered parent material; B/C \\
\hline TP22: & $0-20$ & Loam; 10YR4/2; friable; granular; roots present, $<2$ mm; Ap \\
\hline \multirow[t]{2}{*}{ 18L $04754878475144 ; 3539$} & $20-62$ & Sandy loam; 10YR4/3; friable; blocky; 5-10\% charcoal, $1-2 \mathrm{~cm}$; A2 \\
\hline & $62+$ & Silt loam with abundant fine tonalite fragments; 10YR4/2; extra firm; massive; very weathered parent material; B/C \\
\hline
\end{tabular}

and $\left.P_{\text {in }}=7.85-892.61 \mu \mathrm{g} / \mathrm{ml}\right)$. This is in common with the results from previous research done in the Andes, with the exception of the higher values associated with the use of P-rich fertiliser (Eash, 1989; Goodman-Elgar, 2002; Sandor and Eash, 1991, 1995). Downslope, profile averages of $\mathrm{P}_{\text {org }}$ decreased between Rows 6 and 5, from 157.16 to $89.77 \mu \mathrm{g} / \mathrm{ml}$, then increased to Row 1 at $402.25 \mu \mathrm{g} / \mathrm{ml}$.

\subsection{Micromorphological analysis}

Micromorphological analysis was undertaken on samples from the main indicative stratigraphic horizons represented in Test Pits 1, 2, 4 and 5 (see Table 7; Fig. 5). The make-up of the terrace deposits in Test Pit 1 exhibited a poorly sorted, apedal, sandy/silt loam fabric with grains found in all orientations (Fig. 6a). This soil had once contained a greater organic component as indicated by the vughy nature of the soil fabric (Stolt and Lindbo, 2010), but was neither particularly humic nor affected by the secondary formation of amorphous sesquioxides. Similar material continues to be evident down-profile until the weathered tonalite bedrock material is encountered (Fig. 6b). There is no indication of a buried soil present even though this had been hinted at in the field.
The terrace make-up in Test Pit 2 was very similar to that observed in Test Pit 1. At the base of the test pit there was a similar sandy/silt loam without much humic or amorphous iron staining, but it did exhibit hints of a small blocky ped structure and occasional aggregates, and a few coatings of pure to dusty (silty) clay in the groundmass (Fig. 6c). This is suggestive of a possible weathered B or Bw (cambic) horizon remnant of a buried soil (Kuhn et al., 2010).

Test Pit 3 was not sampled as there was only c. $15 \mathrm{~cm}$ of present day topsoil over the weathered bedrock. Test Pit 4 was also shallow with only $20 \mathrm{~cm}$ of modern topsoil over a possible buried soil that was similar to that in the base of Test Pit 2 (Fig. 6d). The basal horizon of Test Pit 5 exhibited a similar fabric to the other possible old land surface in Test Pit 2, a sandy/silt loam, but in this case it had common micro-charcoal and occasional void in-fills of phytolith-rich ash (Fig. 6e). The latter are suggestive of deliberate additions of organic midden-derived material to the soil as fertiliser that have worked their way down-profile in the pore-soil water system and by soil faunal mixing (Stolt and Lindbo, 2010), but do not appear to be a common feature of these terrace soils as observed in the other test pits.

The make-up material of the stone terraces of system B on the downhill slopes to the west of the Sangayaico site complex was much thinner

Table 3

The analytical methods used in this study.

\begin{tabular}{|c|c|c|}
\hline Analysis & Method & Reference \\
\hline pH \& electrical conductivity & $1: 1$ soil slurry & $\begin{array}{l}\text { Corwin and Lesch (2003); Deetz and Dethlefsen (1963); Rhoades et } \\
\text { al., 1999; Smith et al. (1996); Thomas (1996) }\end{array}$ \\
\hline Soil moisture content & $30 \mathrm{~g}$ at $100{ }^{\circ} \mathrm{C}$ in oven for $48 \mathrm{~h}$ & Patriquin (2003); Pierzynski et al. (2005) \\
\hline Organic and inorganic carbon & Loss-on-ignition at 500 and $900{ }^{\circ} \mathrm{C}$ & Heiri et al. (2001); Schumacher (2002) \\
\hline Particle size analysis & $\begin{array}{l}\text { Wet sieve and Malvern Laser Mastersizer, dispersed in targeted } \mathrm{pH} \\
\text { solution }\end{array}$ & $\begin{array}{l}\text { Gee and Bauder (1986); Gee and Or (2002); Keller and Gee (2006); } \\
\text { Konert and Vandenberghe (1997); Nanavati et al. (2013) }\end{array}$ \\
\hline Phosphorus determination & Ignition and extraction with $\mathrm{H}_{2} \mathrm{SO}_{4}$ & Bowman (1988); Holliday and Gartner, 2007; Kuo (1996) \\
\hline Soil micromorphology & $\begin{array}{l}\text { Impregnation under vacuum with crystic resin; when hardened - } \\
\text { cutting, polishing and mounting slice on large format glass slide, } \\
\text { then grinding to } 25-30 \mu \mathrm{m} \text { on Brot thin section machine, and } \\
\text { cover-slipping }\end{array}$ & $\begin{array}{l}\text { Courty et al. (1989); Bullock et al. (1985); Murphy (1986); Stoops } \\
\text { (2003) }\end{array}$ \\
\hline
\end{tabular}


Table 4

Summary of the bulk physical results for pH, electrical conductivity, soil moisture content, organic/inorganic carbon, total carbon and nitrogen, soil texture and phosphorus.

\begin{tabular}{|c|c|c|c|c|c|c|c|}
\hline Analysis & Method & $\begin{array}{l}\text { Number of } \\
\text { samples }\end{array}$ & Mean $^{\mathrm{a}}$ & Range & $\mathrm{SD}(\sigma)$ & $\begin{array}{l}\text { Limits of } \\
\text { determination }\end{array}$ & Reference \\
\hline Electrical conductivity & $1: 1$ soil slurry & 58 & $57.8 \mu \mathrm{S} / \mathrm{m}$ & $\begin{array}{l}152.2 \mu \mathrm{S} / \mathrm{m} \\
(84.10 \%)\end{array}$ & 31.42 & $1 \%$ & $\begin{array}{l}\text { Corwin and Lesch (2003); Deetz } \\
\text { and Dethlefsen (1963); Rhoades } \\
\text { et al., 1999; Thomas (1996) }\end{array}$ \\
\hline Soil moisture content & $30 \mathrm{~g}$ in $100^{\circ} \mathrm{C}$ oven for $48 \mathrm{~h}$ & 58 & $2.60 \%$ & $5.19 \%$ & 1.58 & $1 \%$ & Pierzynski et al. (2005) \\
\hline \multirow{2}{*}{$\begin{array}{l}\text { Organic and inorganic } \\
\text { matter content }\end{array}$} & LOI $500{ }^{\circ} \mathrm{C}$ & 58 & $5.95 \%$ & $8.79 \%$ & 1.45 & \multirow[t]{2}{*}{$1 \%$} & \multirow[t]{2}{*}{ Heiri et al. (2001) } \\
\hline & LOI $900{ }^{\circ} \mathrm{C}$ & 58 & $0.81 \%$ & $0.90 \%$ & 0.24 & & \\
\hline \multirow{2}{*}{$\begin{array}{l}\text { Organic and nitrogen } \\
\text { content }\end{array}$} & Dry combustion in a & 58 & $C=1.19 \%$ & $4.09 \%$ & 0.81 & \multirow[t]{2}{*}{$0.5 \%$} & \multirow{2}{*}{$\begin{array}{l}\text { Heiri et al. (2001); Mulvaney } \\
\text { (1996) }\end{array}$} \\
\hline & LecoTruspec CN Analyser & & $\mathrm{N}=0.11 \%$ & $0.32 \%$ & 0.07 & & \\
\hline \multirow[t]{3}{*}{ Soil texture } & Wet sieve and Malvern Laser & 55 & Sand $=54.75 \%$ & $39.28 \%$ & 8.15 & \multirow[t]{3}{*}{$0.5 \%{ }^{\mathrm{b}}$} & Gee and Bauder (1986); Gee and \\
\hline & Mastersizer, dispersed in & & Silt $=32.85 \%$ & $21.55 \%$ & 5.03 & & Or (2002); Keller and Gee (2006); \\
\hline & targeted $\mathrm{pH}$ solution & & Clay $=11.44 \%$ & $16.34 \%$ & 2.95 & & $\begin{array}{l}\text { Konert and Vandenberghe } \\
\text { (1997); Nanavati et al. (2013) }\end{array}$ \\
\hline \multirow[t]{3}{*}{ P-determination } & $\begin{array}{l}\text { Ignition and extraction with } \\
\mathrm{H}_{2} \mathrm{SO}_{4}\end{array}$ & 32 & $\begin{array}{l}\text { Ptot }=788.17 \mu \mathrm{g} / \mathrm{ml} \\
(100 \%)\end{array}$ & $1075.87 \mu \mathrm{g} / \mathrm{ml}$ & 221.45 & \multirow[t]{3}{*}{$4 \%^{\mathrm{c}}$} & Bowman (1988) \\
\hline & & & $\begin{array}{l}\text { Pin }=568.29 \mu \mathrm{g} / \mathrm{ml} \\
(72 \%)\end{array}$ & $\begin{array}{l}867.33 \mu \mathrm{g} / \mathrm{ml} \\
(73 \%)\end{array}$ & 203.88 & & Rhoades et al., 1999, \\
\hline & & & $\begin{array}{l}\text { Porg }=218.88 \\
\mu \mathrm{g} / \mathrm{ml}(28 \%)\end{array}$ & $\begin{array}{l}901.68 \mu \mathrm{g} / \mathrm{ml} \\
(67 \%)\end{array}$ & 163.70 & & Kuo (1996) \\
\hline
\end{tabular}

\footnotetext{
a Mean pH calculated by averaging $\mathrm{H}+$ concentrations.

b Sample and sample preparation dependent.

c Estimation based on Bowman, 1988.
}

than had been expected, ranging in thickness from c. 56-67 cm. In Test Pit 5 there was a hint of an old land surface present in the basal c. $16 \mathrm{~cm}$ of the profile, but not in Test Pits 21 and 22. The terrace deposits are consistently composed of a poorly sorted mixture of very fine to fine sandsized quartz and tonalite fragments with a humic silt fine fraction in between (Fig. 6a). The thin surviving buried soil/old land surface is composed of a similar fabric but was less porous, somewhat better sorted, with a weakly developed blocky ped structure, occasional pure to dusty clay in the groundmass, and a greater included humic component which also comprised plant derived ash.

\section{Discussion}

Soil micromorphological analysis suggests that reasonably well defined old land surfaces/buried soils were only present in Test Pit 2 (and possibly Test Pits 7, 10-17, 19 and 20) in Terrace System A and Test Pit 5 in Terrace System B. Where buried soils were evident, they were thin and patchy and poorly developed, with only minor illuviation of silts and clays evident in the sandy/silt loam.

The majority of the $c .25-75 \mathrm{~cm}$ terrace build-up was composed of a similar sandy/silt loam soil material, but mainly without illuviation features, intermixed with common to abundant tonalite rock fragments of varying sizes. In many respects the terrace build-up material resembles a depleted eluvial Eb horizon, with increased coarser, sand-sized and stone-sized components. The lack of variability in clay content down-profile is likely due to the eluviation of clays from the A horizon (Sandor and Eash, 1995). This points to a combination of lateral and down-profile soil flushing caused by introduced water from irrigation, as well as physical mixing processes associated with past arable use of the terraces, the incorporation of organic matter and strong soil faunal activity, and exposure and weathering of the tonalite bedrock in the upper part of each terrace. It should also be noted that in the Andean sierra, soils are exposed to diurnal freeze-thaw variations that when combined with intense solar radiation and dramatic differences in seasonal and annual variability in rainfall, accelerate the soil mixing processes and the weathering of the parent material and downslope erosion processes throughout the soil system (Contreras, 2010; Goodman-Elgar, 2008; Van Vliet-Lanoe, 2010).

The basal terrace soils are essentially stabilised versions of the terrace make-up material above. Any real presence and depth of older (earlier Holocene) soils are ostensibly missing beneath the terrace systems investigated, but geoarchaeological investigations by $\mathrm{C}$. French as part of the same overall project have discerned argillic fine sandy clay loam soils present in the Olaya valley about $200 \mathrm{~m}$ and $2 \mathrm{~km}$ upstream of Sangayaico. Thus it is possible that these argillic soils (or luvisols) were once more widespread in the catchment, but have generally changed beyond recognition quite rapidly, first to colluvial sandy loams and then to terrace accumulations of rubbly sandy/silt loam over shallow, weakly developed, often truncated, A-B/C or A-B-B/C/C cambisol or leptosol-type soils. Down-slope erosion and associated soil truncation prior to the establishment of the terraces would have been a real consideration in causing this soil change, but are almost impossible to quantify, and it is impossible to rule out previous agricultural activities on the slopes also contributing to this apparent major change in soil type and its survival.

The chronology of these changes is much harder to ascribe with any real accuracy. Certainly other examples of Andean terrace systems are fully developed by about 1300 years ago (Beach et al., 2015), and it is reasonable to assume that the terrace systems on the slopes adjacent to Sangayaico are at least associated with the settlements that are dated there to cal AD 1122-1527. Either way, there is a strong probability that the soil-scape on the hillsides has been highly modified by the past establishment of the terrace system(s), perhaps over no more than the past $800-900$ years or so. The whole soil complex is relatively young and under-developed. Nonetheless, these terrace soils appear to have been well managed, essentially through the repeated addition of organic matter.

Arable cropping would have continued to deplete the nutrient and organic matter levels of these terrace soils. This would have occurred despite the regular introduction of water carried down valley along-slope by the main stone irrigation channels, fed by spring/ river water from the pampa zone and sluices letting the water downslope into each set of terraces (Denevan, 2001), the continuing deliberate addition of organic matter from the turning in of harvested crops, pastoral herds being kept on these fields in-between cropping seasons and any deliberate additions of household midden debris. To the detriment of the wider soil system on the valley sides, irrigation and rainfall combined would have encouraged the flushing of fines and nutrients from these soils down-profile and down-slope as lateral flushes, possibly counter-acting the moisture retention aspect of the thickened terrace soils themselves. Consequently long fallow 
Table 5

Bulk sample results for $\mathrm{pH}, \mathrm{C}: \mathrm{N}$ ratio, particle size distribution and phosphorus.

\begin{tabular}{|c|c|c|c|c|c|c|c|c|c|c|c|c|c|c|c|c|c|c|c|c|}
\hline \multirow[b]{2}{*}{ Sample \# } & \multirow[b]{2}{*}{ Row } & \multirow[b]{2}{*}{ Column } & \multirow[b]{2}{*}{ Layer } & \multirow[b]{2}{*}{$\begin{array}{c}\text { Depth } \\
(\mathrm{cm})\end{array}$} & \multicolumn{2}{|c|}{ Electrode } & \multirow{2}{*}{$\begin{array}{c}\text { SMC } \\
\mathrm{H}_{2} \mathrm{O} \\
(\%)\end{array}$} & & & Leco & C:N Ana & & & $\begin{array}{l}\text { Irticle S } \\
\text { tibution }\end{array}$ & & Pho & horus ( $\mu \mathrm{g}$ & $\mathrm{mL})$ & $\mathrm{Pho}$ & $\begin{array}{l}\text { horus } \\
\text { 6) }\end{array}$ \\
\hline & & & & & $\mathrm{pH}$ & $\begin{array}{c}\mathrm{EC} \\
(\mu \mathrm{S} / \mathrm{m})\end{array}$ & & $\mathrm{OM}$ & $\mathrm{CaCO}_{3}$ & $\mathrm{~N}(\%)$ & $\mathrm{C}(\%)$ & $\mathrm{C}: \mathrm{N}$ & Clay & Silt & Sand & $P_{\text {tot }}$ & $P_{\text {in }}$ & $\mathrm{P}_{\mathrm{org}}$ & $\mathrm{P}_{\text {in }}$ & $P_{\text {org }}$ \\
\hline TP-20 A & 6 & A & I & 5 & 6.63 & 76.5 & 0.86 & 5.13 & 0.60 & 0.13 & 1.66 & 12.79 & 9.96 & 30.64 & 61.69 & & & & & \\
\hline TP-20 Eb & 6 & A & II & 25 & 6.38 & 40.2 & 1.26 & 4.01 & 0.56 & 0.08 & 0.75 & 9.97 & 8.41 & 28.36 & 66.10 & & & & & \\
\hline $\mathrm{TP}-20 \mathrm{bA} 2$ & 6 & A & III & 40 & 6.42 & 57.5 & 1.47 & 4.58 & 0.57 & 0.08 & 1.01 & 12.30 & 7.54 & 29.72 & 65.61 & & & & & \\
\hline $\mathrm{TP}-20 \mathrm{~B} / \mathrm{C}$ & 6 & A & IV & 48 & 6.66 & 51.8 & 1.65 & 3.68 & 0.65 & 0.02 & 0.33 & 16.59 & 7.57 & 29.38 & 65.88 & & & & & \\
\hline TP-16 A & 5 & A & I & 2 & 5.97 & 178.8 & 1.14 & 5.30 & 0.51 & 0.12 & 1.38 & 11.19 & 11.06 & 31.97 & 58.93 & & & & & \\
\hline TP-16 Eb & 5 & A & II & 30 & 6.31 & 37.8 & 1.27 & 4.48 & 0.49 & 0.10 & 1.04 & 10.51 & 10.22 & 29.25 & 62.96 & & & & & \\
\hline TP-16 bA2 & 5 & A & III & 40 & 6.77 & 44.7 & 2.97 & 3.95 & 0.74 & 0.02 & 0.27 & 15.94 & 6.45 & 28.26 & 68.22 & & & & & \\
\hline TP-16 bA2 & 5 & A & & 45 & 6.67 & 37.8 & 1.67 & 2.53 & 0.56 & 0.02 & 0.25 & 14.91 & & & 57.56 & & & & & \\
\hline TP-16 B/C & 5 & A & & 57 & 6.68 & 41.3 & 1.21 & 2.10 & 0.56 & 0.03 & 0.34 & 9.84 & & & 62.02 & & & & & \\
\hline TP-16 bEb & 5 & A & IV & 55 & 6.87 & 43.5 & 2.61 & 3.64 & 0.66 & 0.03 & 0.34 & 9.89 & 7.59 & 29.04 & 66.05 & & & & & \\
\hline TP-14 A & 4 & A & I & 5 & 6.48 & 64.6 & 1.47 & 5.11 & 0.65 & 0.12 & 1.22 & 10.02 & 10.65 & 32.61 & 58.56 & & & & & \\
\hline TP-14 Eb & 4 & A & II & 15 & 6.81 & 52.5 & 2.08 & 4.97 & 0.82 & 0.09 & 1.00 & 10.59 & 10.21 & 32.66 & 58.84 & & & & & \\
\hline TP-14 Eb & 4 & A & III & 25 & 6.63 & 28.3 & 3.01 & 4.44 & 0.80 & 0.06 & 0.60 & 9.84 & 11.46 & 31.06 & 59.36 & & & & & \\
\hline TP-14 bA2 & 4 & A & IV & 30 & 6.55 & 26.6 & 4.42 & 3.79 & 1.12 & 0.04 & 0.33 & 8.12 & 12.55 & 33.84 & 55.04 & & & & & \\
\hline TP-2 A & 3 & A & I & 5 & 6.79 & 106.0 & 1.36 & 5.75 & 0.64 & 0.15 & 1.58 & 10.79 & 10.60 & 32.06 & 59.44 & & & & & \\
\hline TP-2 Eb & 3 & A & II & 15 & 6.71 & 50.5 & 1.45 & 4.44 & 0.60 & 0.09 & 0.91 & 9.57 & 10.51 & 31.07 & 60.41 & & & & & \\
\hline TP-2 bA2 & 3 & A & III & 30 & 6.8 & 56.5 & 2.01 & 4.58 & 0.72 & 0.05 & 0.48 & 8.70 & 8.82 & 26.29 & 67.85 & & & & & \\
\hline TP-2 B/C & 3 & A & IV & 40 & 6.64 & 47.0 & 2.50 & 4.02 & 0.65 & 0.03 & 0.39 & 12.37 & 8.84 & 25.05 & 69.70 & & & & & \\
\hline TP-9 A & 2 & A & I & 2 & 6.47 & 80.3 & 1.58 & 6.33 & 0.71 & 0.12 & 1.27 & 10.34 & 10.99 & 30.99 & 60.08 & & & & & \\
\hline TP-9 Eb & 2 & A & II & 8 & 6.57 & 54.2 & 1.81 & 5.16 & 0.76 & 0.09 & 0.89 & 9.37 & 11.83 & 32.62 & 57.22 & & & & & \\
\hline TP-9 B/C & 2 & A & III & 10 & 6.52 & 35.3 & 2.96 & 5.10 & 1.46 & 0.02 & 0.37 & 15.77 & 13.97 & 40.32 & 46.20 & & & & & \\
\hline TP-7 A & 1 & A & I & 3 & 6.26 & 38.2 & 1.74 & 6.20 & 1.01 & 0.13 & 1.41 & 10.78 & 15.30 & 40.36 & 44.87 & & & & & \\
\hline TP-7 Eb & 1 & A & II & 5 & 6.03 & 54.6 & 1.91 & 5.82 & 1.03 & 0.11 & 0.90 & 8.18 & 14.83 & 37.23 & 48.85 & & & & & \\
\hline TP-7 bA2 & 1 & A & III & 20 & 6.06 & 56.7 & 2.40 & 5.88 & 0.92 & 0.10 & 0.93 & 9.61 & 15.23 & 38.19 & 47.52 & & & & & \\
\hline TP-7 B/C & 1 & $\mathrm{~A}$ & IV & 40 & 6.18 & 44.3 & 6.05 & 7.17 & 1.13 & 0.06 & 0.53 & 8.35 & 19.29 & 42.37 & 38.78 & & & & & \\
\hline TP-18 A & 6 & $\mathrm{C}$ & I & 5 & 5.33 & 52.8 & 1.98 & 10.89 & 0.74 & 0.34 & 4.39 & 12.95 & 14.94 & 39.68 & 46.27 & 899.28 & 611.15 & 288.13 & 68 & 32 \\
\hline TP-18 Eb & 6 & $\mathrm{C}$ & II & 15 & 6.17 & 40.8 & 1.40 & 5.31 & 0.79 & 0.10 & 1.18 & 11.78 & 10.10 & 32.53 & 59.33 & 775.31 & 710.33 & 64.98 & 92 & 8 \\
\hline TP-18 B/C & 6 & $\mathrm{C}$ & III & 25 & 6.42 & 57.4 & 1.41 & 4.77 & 0.63 & 0.08 & 0.88 & 11.33 & & & 30.42 & 875.09 & 683.77 & 191.31 & 78 & 22 \\
\hline TP-18 B/C & 6 & $\mathrm{C}$ & IV & 27 & 6.68 & 67.7 & 1.73 & 4.34 & 0.72 & 0.04 & 0.48 & 12.35 & 11.17 & 30.36 & 60.31 & 974.42 & 890.21 & 84.21 & 91 & 9 \\
\hline TP-17 A & 5 & $\mathrm{C}$ & I & 2 & 6.25 & 83.9 & 1.45 & 6.53 & 0.79 & 0.15 & 1.75 & 11.73 & 13.79 & 40.63 & 46.40 & 874.83 & 785.76 & 89.06 & 90 & 10 \\
\hline TP-17 Eb & 5 & $\mathrm{C}$ & II & 15 & 6.23 & 40.7 & 2.58 & 6.04 & 0.93 & 0.10 & 1.18 & 11.42 & 10.52 & 35.74 & 54.97 & 774.61 & 564.22 & 210.39 & 73 & 27 \\
\hline TP-17 bA2 & 5 & $\mathrm{C}$ & III & 30 & 6.37 & 30.2 & 3.77 & 5.16 & 0.99 & 0.05 & 0.49 & 9.07 & 12.53 & 39.56 & 48.89 & 599.40 & 570.96 & 28.44 & 95 & 5 \\
\hline TP-17 B/C & 5 & $\mathrm{C}$ & IV & 40 & 6.29 & 28.6 & 3.85 & 5.40 & 1.38 & 0.02 & 0.31 & 17.74 & 8.57 & 26.19 & 67.85 & 499.60 & 468.41 & 31.19 & 94 & 6 \\
\hline TP-1 A & 4 & $\mathrm{C}$ & I & 3 & 6.12 & 81.7 & 1.91 & 8.80 & 0.56 & 0.19 & 2.24 & 11.53 & 13.47 & 37.99 & 49.80 & 874.13 & 713.50 & 160.63 & 82 & 18 \\
\hline TP-1 Eb & 4 & $\mathrm{C}$ & II & 15 & 6.34 & 54.1 & 1.56 & 5.26 & 0.48 & 0.09 & 0.93 & 9.93 & 10.15 & 32.30 & 59.54 & 824.59 & 584.41 & 240.17 & 71 & 29 \\
\hline TP-1 bA2 & 4 & $\mathrm{C}$ & III & 40 & 6.42 & 53.3 & 11.43 & 4.86 & 0.54 & 0.07 & 0.79 & 10.95 & 10.86 & 37.07 & 53.20 & 674.87 & 564.03 & 110.84 & 84 & 16 \\
\hline TP-1 B/C & 4 & $\mathrm{C}$ & IV & 50 & 6.6 & 54.4 & 2.07 & 4.72 & 0.82 & 0.03 & 0.30 & 10.80 & 8.36 & 26.72 & 68.06 & 700.63 & 637.92 & 62.71 & 91 & 9 \\
\hline TP-11 A & 3 & $\mathrm{C}$ & I & 3 & 6.28 & 74.6 & 1.59 & 6.30 & 0.59 & 0.15 & 1.51 & 9.99 & 13.40 & 37.61 & 50.14 & 950.38 & 685.91 & 264.47 & 72 & 28 \\
\hline $\mathrm{TP}-11 \mathrm{~Eb}$ & 3 & $\mathrm{C}$ & II & 15 & 6.16 & 35.6 & 1.94 & 5.01 & 0.59 & 0.09 & 0.93 & 9.95 & 11.28 & 32.89 & 57.52 & 875.61 & 459.28 & 416.33 & 52 & 48 \\
\hline TP-11 bA2 & 3 & $\mathrm{C}$ & III & 30 & 6.34 & 39.6 & 1.77 & 4.24 & 0.50 & 0.05 & 0.50 & 10.48 & 9.42 & 29.63 & 63.23 & 750.45 & 433.03 & 317.42 & 58 & 42 \\
\hline $\mathrm{TP}-11 \mathrm{~B} / \mathrm{C}$ & 3 & $\mathrm{C}$ & IV & 36 & 6.43 & 38.5 & 1.82 & 4.59 & 0.51 & 0.05 & 0.53 & 9.95 & 11.36 & 31.69 & 58.90 & 749.48 & 432.60 & 316.87 & 58 & 42 \\
\hline TP-8 A & 2 & $\mathrm{C}$ & I & 3 & 6.21 & 158.5 & 1.72 & 7.53 & 0.97 & 0.23 & 2.76 & 12.25 & 12.70 & 39.30 & 49.02 & 824.59 & 483.15 & 341.44 & 59 & 41 \\
\hline TP-8 Eb & 2 & $\mathrm{C}$ & II & 20 & 6.18 & 40.4 & 2.32 & 5.60 & 0.99 & 0.11 & 1.08 & 9.59 & 12.92 & 38.91 & 49.16 & 624.69 & 435.04 & 189.65 & 70 & 30 \\
\hline TP-8 B/C & 2 & $\mathrm{C}$ & III & 23 & 6.23 & 43.7 & 4.48 & 6.33 & 1.13 & 0.05 & 0.41 & 8.84 & 13.39 & 35.93 & 51.85 & 224.91 & 7.85 & 217.06 & 3 & 97 \\
\hline TP-13 A & 1 & $\mathrm{C}$ & I & 3 & 6.63 & 86.0 & 911. & 6.12 & 0.85 & 0.11 & 1.18 & 10.54 & 12.29 & 34.89 & 54.11 & 600.24 & 458.76 & 141.48 & 76 & 24 \\
\hline TP-13 Eb & 1 & C & II & 15 & 6.4 & 42.8 & 2.42 & 5.38 & 0.99 & 0.10 & 0.91 & 9.06 & 12.21 & 34.74 & 54.43 & 650.46 & 410.26 & 240.20 & 63 & 37 \\
\hline TP-13 bA2 & 1 & $\mathrm{C}$ & III & 20 & 6.46 & 40.0 & 3.63 & 5.34 & 1.03 & 0.07 & 0.61 & 9.19 & 12.03 & 34.09 & 55.29 & 1300.78 & 363.03 & 937.75 & 28 & 72 \\
\hline $\mathrm{TP}-13 \mathrm{~B} / \mathrm{C}$ & 1 & $\mathrm{C}$ & IV & 45 & 6.47 & 37.2 & 3.83 & 5.96 & 1.55 & 0.03 & 0.34 & 12.29 & 22.79 & 37.62 & 40.15 & 299.97 & 10.40 & 289.57 & 3 & 97 \\
\hline TP-5 A & 9 & $\mathrm{D}$ & I & 10 & 6.84 & 175.0 & 2.34 & 10.54 & 0.47 & 0.33 & 3.85 & 11.74 & 15.48 & 42.43 & 42.79 & 974.81 & 665.64 & 309.16 & 68 & 32 \\
\hline TP-5 Eb & 9 & D & II & 40 & 6.74 & 64.6 & 3.02 & 8.09 & 0.87 & 0.20 & 2.37 & 11.79 & 15.94 & 44.16 & 40.45 & 724.57 & 567.40 & 157.17 & 78 & 22 \\
\hline TP-5 bA2 & 9 & $\mathrm{D}$ & III & 50 & 6.88 & 42.4 & 4.27 & 4.85 & 0.76 & 0.07 & 0.64 & 9.55 & 13.41 & 34.25 & 53.57 & 824.59 & 1201.21 & -376.62 & -46 & 146 \\
\hline TP-5 B/C & 9 & D & IV & 56 & 6.85 & 44.9 & 2.19 & 5.14 & 0.82 & 0.05 & 0.49 & 9.07 & 14.40 & 34.33 & 52.61 & 675.68 & 588.24 & 87.43 & 87 & 13 \\
\hline TP-21 Ap & 8 & D & I & 5 & 6.3 & 49.1 & 1.78 & 5.73 & 0.68 & 0.13 & 1.33 & 10.03 & 11.72 & 37.89 & 51.57 & 799.36 & 611.22 & 188.14 & 76 & 24 \\
\hline TP-21 A2 & 8 & D & II & 60 & 6.88 & 55.1 & 2.07 & 5.10 & 0.71 & 0.08 & 0.79 & 9.51 & 12.94 & 34.89 & 53.51 & 1174.30 & 892.61 & 281.69 & 76 & 24 \\
\hline TP-21 B/C & 8 & D & III & 67 & 7.19 & 47.1 & 2.19 & 5.14 & 0.82 & 0.04 & 0.48 & 11.65 & 13.27 & 38.02 & 49.72 & 674.60 & 562.11 & 112.49 & 83 & 17 \\
\hline TP-22 Ap & 7 & D & I & 15 & 6.59 & 48.4 & 1.79 & 5.56 & 0.75 & 0.12 & 1.09 & 8.84 & 14.90 & 43.90 & 41.73 & 924.82 & 712.30 & 212.52 & 77 & 23 \\
\hline TP-22 A2 & 7 & D & II & 40 & 6.62 & 53.8 & 1.84 & 6.77 & 0.85 & 0.17 & 1.80 & 10.83 & 15.20 & 46.60 & 45.59 & 950.00 & 789.16 & 160.84 & 83 & 17 \\
\hline $\mathrm{TP}-22 \mathrm{~B} / \mathrm{C}$ & 7 & $\mathrm{D}$ & III & 62 & 6.84 & 45.7 & 1.62 & 4.79 & 0.78 & 0.09 & 0.82 & 9.51 & 14.24 & 39.40 & 47.24 & 1125.11 & 864.63 & 260.48 & 77 & 23 \\
\hline & & & & & 6.48 & 57.1 & 2.39 & 5.42 & 0.78 & 0.09 & 1.03 & 10.91 & 12.07 & 34.64 & 54.75 & 789.28 & 588.44 & 200.84 & 67 & 33 \\
\hline & & & & & 1.86 & 152.2 & 5.19 & 8.79 & 0.90 & 0.32 & 3.55 & 4.61 & 12.54 & 9.36 & 8.73 & 949.39 & 884.76 & 909.31 & 92 & 92 \\
\hline & & & $\begin{array}{r}\text { Lin } \\
\text { Deter }\end{array}$ & $\begin{array}{l}\text { ts of } \\
\text { nation }\end{array}$ & $0.1 \%$ & $1 \%$ & & $1 \%$ & & & $0.5 \%$ & & & $0.5 \%$ & & & & $4 \%$ & & \\
\hline
\end{tabular}

recovery periods of several years would have been required to maintain a reasonable fertility in these soils as well as regular burning of the fields after each growing season (as practised today). Even then, recovery of fertility would have been slow and any real soil development unlikely, a feature which is recognised today despite much of this highland area being abandoned and largely unused. 
Table 6

Trends in soil characteristics down-profile and down-slope for each terrace system.

\begin{tabular}{|c|c|c|c|c|c|c|}
\hline $\begin{array}{l}\text { Terrace } \\
\text { system }\end{array}$ & $\begin{array}{l}\text { Terrace } \\
\text { build-up/buried } \\
\text { soil }\end{array}$ & $\mathrm{pH}$ & Soil moisture & Organic content & Phosphorus & $\begin{array}{l}\text { Micromorphology \& } \\
\text { particle size }\end{array}$ \\
\hline A, & $\begin{array}{l}25-60 \mathrm{~cm} \text { terrace } \\
\text { build-up; buried bA2 } \\
\text { in TP } 1-4,7,10-17 \text { \& } \\
19-20\end{array}$ & $\begin{array}{l}\text { Weakly acidic to } \\
\text { neutral; range of } \\
5.3-6.87\end{array}$ & $\begin{array}{l}\text { Low, variable; range } \\
\text { of } 0.86-6 \% ; 1 \text { to } 4 \% \\
\text { increase lower in } \\
\text { profiles }\end{array}$ & $\begin{array}{l}\text { Range from } 2 \text { to } 7 \% \text {; general } \\
\text { decrease of }<1 \% \text { down-profile; } \\
10.89 \% \text { high in A of TP18 \& 8.8\% } \\
\text { in A of TP1 }\end{array}$ & $\begin{array}{l}\text { NO trend, variable, low } \\
\text { to moderate enhanced, } \\
300-1300 \mu \mathrm{g} / \mathrm{ml} \text {; lower } \\
\text { values can be in Eb of } \\
\text { terrace make-up }\end{array}$ & $\begin{array}{l}\text { Increased clay and silt } \\
\text { in Row } 1 \text {, especially in } \\
\text { bA2; dusty clay in } \\
\text { groundmass } \\
\text { in bA2 in TP } 1,2 \& 4\end{array}$ \\
\hline A, downslope & As above & As above & $\begin{array}{l}\text { Low, variable; range } \\
\text { of } 1-6 \% ; 11.43 \% \\
\text { high in Eb of TP1 } \\
\text { mid-slope }\end{array}$ & Steady to mainly decreasing & $\begin{array}{l}\text { As above; averages } \\
\text { decrease between Rows } 6 \\
\& 5 \text {, but increases in Row } 1\end{array}$ & As above \\
\hline B, & $\begin{array}{l}55-67 \mathrm{~cm} \text { terrace } \\
\text { build-up; buried bA2 } \\
\text { in TP5 only }\end{array}$ & $\begin{array}{l}\text { Neutral to weakly } \\
\text { basic; range of } \\
6.3-7.19 \text {; slightly more } \\
\text { basic with depth }\end{array}$ & $\begin{array}{l}\text { Low, little variation; } \\
\text { range of } 1.62-4.27 \%\end{array}$ & $\begin{array}{l}\text { Steady at } 4.79-5.73 \% \text {, except } \\
\text { for } 8-10.54 \% \text { in Ah/Eb of TP5 }\end{array}$ & $\begin{array}{l}\text { No trend; generally weak } \\
\text { to moderate enhanced; } \\
\text { note ignore errant values } \\
\text { in Eb of TP5 }\end{array}$ & $\begin{array}{l}\text { Little variation in clay; } \\
\text { minor ash \& very fine } \\
\text { charcoal additions in } \mathrm{Eb} \\
\text { of TP5 }\end{array}$ \\
\hline B, downslope & As above & $\begin{array}{l}\text { Neutral to weakly } \\
\text { basic }\end{array}$ & Low, little variation & As above & As above & $\begin{array}{l}\text { Slightly more sand in } \\
\text { Ap \& Eb of Row } 2\end{array}$ \\
\hline
\end{tabular}

In general the physical characteristics revealed in this study of the Sangayaico terrace system do not show the same clear evidence of soil thickening, modification and long-term fertilisation that was observed by similar studies such as in the Colca Valley of Peru (Eash and Sandor, 1995), nor the distinctive increase in clay illuviation noted in the soils of the Tocotoccasa terrace system in the Chicha-Soras valley (Kemp et al., 2006; Branch et al., 2007). In the Colca study it was observed that A horizons were commonly thicker by c. 30-130 cm, exhibited a lower bulk density (implying a much greater organic content), and the upper horizons were enriched with organic matter. Other studies observed lower pH values, more organic carbon and nitrogen, the addition and inclusion of midden-derived material, and the associated deep translocation and enhancement with phosphorus of the buried B horizons at the base of the terrace profiles (Goodman-Elgar, 2009; Sandor et al., 2007; Wells, 2006). In the Chicha-Soras terrace soil study (Kemp et al., 2006; Branch et al., 2007), the buried upper terrace and surface terrace soils both exhibited an abundance of illuvial clay coatings which were attributed to the weathering, disturbance and down-profile migration of neo-formed clay from the volcanic clasts on site, aggravated by the oscillating arid/humid climate and the repeated input of irrigation water. In contrast, the Sangayaico terraces rarely exhibit over-thickened A horizons, even though there is regularly c. 25$75 \mathrm{~cm}$ of cumulative terrace soil aggradation. The buried B horizons are either thin or not present, and exhibit little sign of enrichment with illuvial clay (or argillic clay) down-profile. Organic matter, nitrogen and phosphorus values are weakly variable and relatively only weakly enhanced, and we know that local farmers did not use artificial fertilisers over the past 50 years or so (from anecdotal accounts of local farmers). This may indicate the lack of recent irrigation as well as a longer-term gradual process of neglect and lack of arable use and fertilisation, and general degradation through hillwash and lateral flush effects through these terrace slopes. Again this is in sharp contrast to the Colca valley study where the farmers appear to have known the exact state and characteristics of their land and how to improve, conserve and husband it successfully (Sandor and Furbee, 1996).

In this Viejo Sangayaico study, no conclusive indicators were observed to more precisely indicate which agricultural strategy may have been employed. The paucity of ceramic and faunal remains and charcoal in and on the terraces themselves, often associated to the removal of household waste (Goodman-Elgar, 2002), and the lack of spikes in soil organic matter and phosphorous $\left(\mathrm{P}_{\text {tot }}, \mathrm{P}_{\mathrm{in}}\right.$, or $\left.\mathrm{P}_{\text {org }}\right)$ averaged across rows, would indicate that midden material was not generally added to the terrace surfaces. Without a natural soil profile to compare to as a control, it is hard to provide quantitative support for an argument attesting to the extent of manuring. But given the similarities of the results presented here to that of Homburg et al. (2005), and the lack of extraordinary peaks in phosphorus as discussed in Sandor and Eash (1991), it is likely that the terrace systems associated with Sangayaico were never extensively fertilised through manuring. Based on ethnographic evidence and field observation, it is probable that manuring was mainly done by grazing animals following the harvest or during a fallow period (De la Vega, 1960; Guillet, 1981, 1987; Zimmerer, 1998). Finally there is no conclusive evidence for seasonal field burning in the Sangayaico terrace systems. Indeed, there was very little charcoal found in the terrace profiles, except for the uppermost levels of the $A_{p}$ horizon in Terrace System B, which probably indicates a recent burning.

As to whether past agricultural strategies have affected long-term soil fertility, the terraces associated with Sangayaico showed little

Table 7

Summary micromorphological descriptions and interpretations.

\begin{tabular}{|c|c|c|}
\hline $\begin{array}{l}\text { Field system test pit and } \\
\text { sample designation }\end{array}$ & Micromorphological description & Interpretation \\
\hline TP1: 3/1, 25-35 cm; 3653 m & $\begin{array}{l}\text { Apedal, porous, poorly sorted, very fine sandy/silt loam with c. } 10 \% \\
\text { tonalite fine stone fragments }\end{array}$ & Modern topsoil of terrace \\
\hline TP1: $3 / 2,42-50 \mathrm{~cm}$ & As above & $\begin{array}{l}\text { Terrace make-up acting as a very poorly developed lower } \\
\text { A/upper B horizon }\end{array}$ \\
\hline TP1: 4, 53-60 cm & $\begin{array}{l}\text { Apedal, stoney, fine sandy/silt loam with up to } 95 \% \text { fine tonalite stone } \\
\text { fragments, }<4 \mathrm{~mm}\end{array}$ & $\begin{array}{l}\text { Mainly weathered bedrock fragments mixed with and soil fabric; } \\
\text { B/C horizon }\end{array}$ \\
\hline TP2: 1, 5-18 cm; $3612 \mathrm{~m}$ & Apedal, porous, poorly sorted, very fine sandy/silt loam & Base of modern topsoil of terrace \\
\hline TP2: $2,20-30 \mathrm{~cm}$ & $\begin{array}{l}\text { Weak sub-angular blocky, sandy/silt loam with few aggregates and } \\
\text { coatings of pure to silty clay in groundmass }\end{array}$ & Possible, very weakly developed Bw of a buried soil \\
\hline TP4: 2, 25-35 cm; $3590 \mathrm{~m}$ & As for TP2, $20-30 \mathrm{~cm}$ & As above \\
\hline TP5: 2, 40-50 cm; $3586 \mathrm{~m}$ & $\begin{array}{l}\text { Apedal, well sorted, sandy/silt loam with common very fine charcoal } \\
\text { and ash inclusions and few ashy void infills }\end{array}$ & $\begin{array}{l}\text { Possible old land surface or B horizon with anthropogenic } \\
\text { additions }\end{array}$ \\
\hline
\end{tabular}




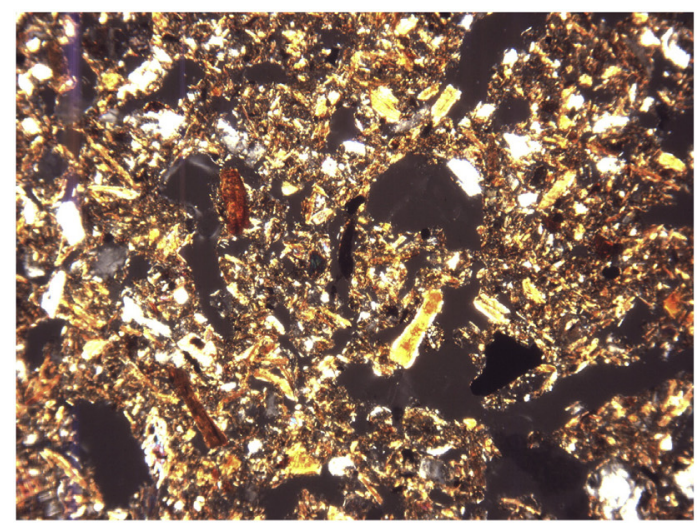

a

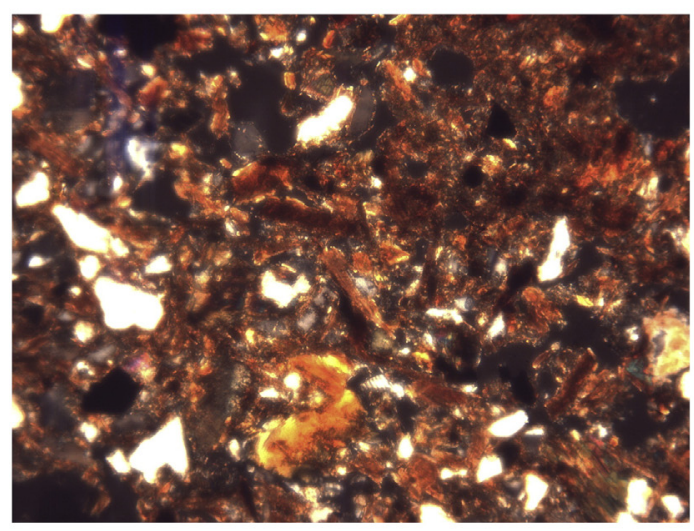

C

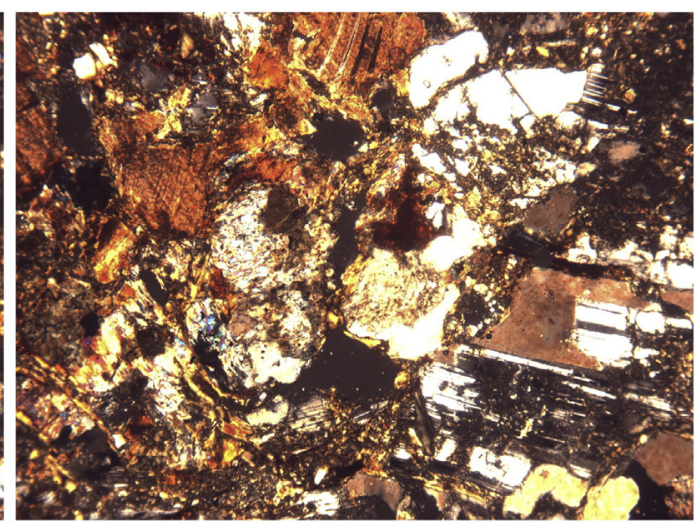

b

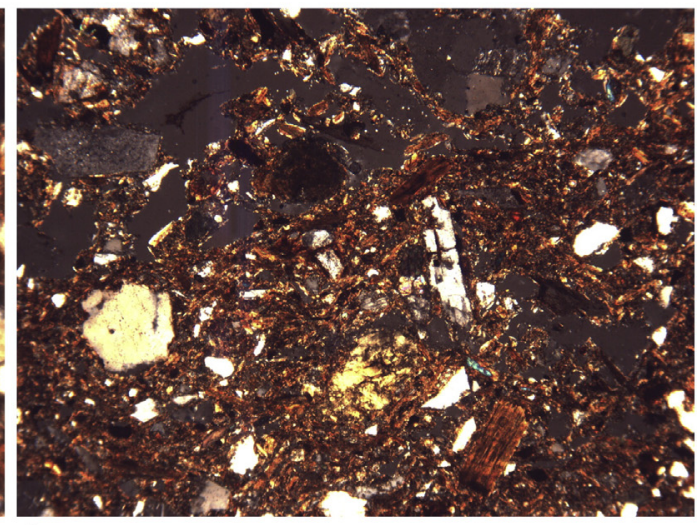

d

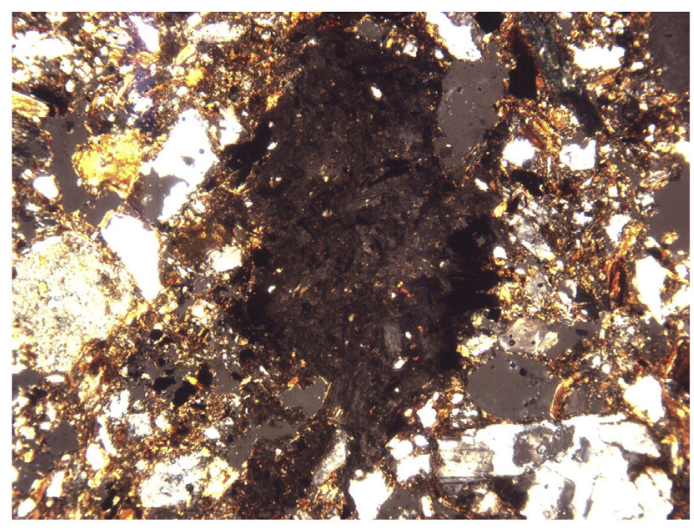

e

Fig. 6. Photomicrographs:

a. Photomicrograph of the poorly sorted, apedal, sandy/silt loam fabric of the terrace make-up, Test Pit 1, sample 3/1 (frame width 4.5 mm; cross polarised light).

b. Photomicrograph of weathered B/C of tonalite fragments, Test Pit 1 , sample 4 (frame width $4.5 \mathrm{~mm}$; cross polarised light).

c. Photomicrograph of the sandy/silt loam fabric with an aggregate of pure clay in the old land surface, Test Pit 2, sample 2/2 (frame width 2.25 mm; cross polarised light).

d. Photomicrograph of the modern ploughsoil/old land surface contact of sandy/silt loam, Test Pit 4, sample 4/2 (frame width 4.5 mm; cross polarised light).

e. Photomicrograph of the phytoliths in the ash infill, Test Pit 5, sample 5/2 (frame width $2.25 \mathrm{~mm}$; cross polarised light).

C. French.

evidence for degradation. But, substantial fallow time and the grazing of animals on the crop stubble would have helped ameliorate this system. Anecdotal conversation with the local farmers working around Sangayaico suggests that the fields remain productive without the use of artificial fertilisers due mainly to the use of a five-year fallow period following a two-three-year growing period. Another important factor in the preservation of the Sangayaico agricultural landscape is the continued use of the chaquitaclla, or Andean foot plough, to turn the soil in the fields. Indeed, using the chaquitaclla greatly reduces the breakdown of beneficial soil aggregates as opposed to mechanised ploughing (Goodman-Elgar, 2008).

It should also be noted that Terrace System A was selected because it appeared to be one of the best preserved terrace systems associated to the Sangayaico site, whilst other terrace systems in the vicinity have fallen into disrepair. The resulting differences between denuded areas and those with better-preserved terracing provides an example of the 
cost of abandoning or neglecting intensive agricultural systems. Such abandonment and neglect was widespread throughout the New World with the arrival of the old-world diseases and the relocation of indigenous populations to live in reducciones, and then again in the last thirty-five to fifty years with massive sierra-to-coast population shifts (Denevan, 2001; Donkin, 1979; Wernke, 2010).

\section{Interpretative discussion and conclusions}

This study has provided the first characterisations of the agrarian soil system in the upper Ica valley surrounding Viejo Sangayaico. The results suggest the relative stability of the terraced systems themselves over the past millennium, but major transformations of the underlying old land surface had already occurred prior to the establishment of the terraced field systems still visible today. Although it requires further investigation and reliable dating, it is very possible that it was erosion, mixing and depletion associated with earlier, pre-Late Intermediate period (or pre- $c$. AD 1100) land-use (for both arable agriculture and pastoralism) that had caused such major soil change on the hill-slopes around Sangayaico. In contrast over the last 900 years or so, local agriculturalists were able to sustainably farm the landscape through the construction of irrigated terraces and the use of crop cycles dependent on longfallowing. This appears to be largely without the extensive use of fertiliser, in contrast to observations in the Colca Valley (Eash and Sandor, 1995; Sandor and Eash, 1991, 1995).

It is suggested that the populations associated with sites throughout the upper and middle Ica River drainage relied on terraces on the mountain slopes as a means of insuring soil stability and conservation. Terraces controlled both landscape erosion and degradation, thus increasing the production area and creating the advantages of a local micro-climate which provided a growing area more amenable to crops normally grown at lower elevation. It is possible that with the arrival of the Spanish and subsequently into the Republican period, the relocation of villagers such as the Sangayaiqueños to mines in the Colonial Period (Bueno Mendoza, 2003; Maldonado Pimentel and Estacio Tamayo, 2012) and subsequent emigration to urban cities (Zimmerer, 1991), the terraces began to fall into disrepair, causing an increase in slope instability and concomitant soil erosion.

Given the relatively low and variable organic matter content and minimal plant macro-nutrients such as phosphorus in the terrace soil systems around Sangayaico, experienced learned knowledge would have been needed to select the appropriate best crop for each area. The amount of forethought in crop selection would have called for a great deal of knowhow about the behaviour of local crops and soils, as has been observed by Sandor and Furbee (1996) for the Colca Canyon. By acknowledging that the conditions for plant growth do not necessarily occur within strictly delimited ecozones or crop range limits but in a more complex mosaic across the landscape, in effect the range of crops can be 'stretched' (Zimmerer, 1999).

Thus strict adherence to models relying on defined ecotones appears to be ill-advised and that using Zimmerer's (1999) "overlapping patchwork" framework approach appears to offer a more holistic and comprehensive explanation of Andean ecology and agricultural land-use. Although the relative instability of the underlying soil properties in this region may hinder specialised cropping, variation in erosion factors would have favoured mixed-cropping systems and allowed a more diverse range of crop production (Zimmerer, 1999). Future palynological studies of the vegetational sequences in the basin mires in the puna upvalley from Sangayaico may shed better light on this suggestion of mixed cropping in due course. Nonetheless, this variability is well documented in Andean soil systems from previous studies (Eash, 1989; Eash and Sandor, 1995; Goodman-Elgar, 2002; Goodman-Elgar, 2008; Kemp et al., 2006; Sandor and Eash, 1991, 1995) as well as at the landscape scale through ecological and human land-use observations (Contreras, 2010; Branch et al., 2007; Zimmerer, 1999). The main reason for this variability is due to the fact that the Andean agro- ecosystem is the result of the coupling of localised climatological, geological and geomorphological processes (Montgomery et al., 2001), shaped, altered and managed by tremendous human endeavour.

\section{Acknowledgements}

The authors would like to thank our hosts, benefactors and collaborators in Peru, particularly Alberto Benavides, Susanna Torres Acres and George Chauca. Funding for the field and laboratory work was provided by the Leverhulme Trust (research grant number RPG-117), a private donation, and the Department of Anthropology, Washington State University (in-kind). Many thanks to Professors Melissa Goodman-Elgar, James Harsh, and Timothy Kohler for advising on the soil fertility and archaeological research aspects completed at Washington State University. We would also like to thank Tonko Rajkovaca of the McBurney Geoarchaeology Laboratory, Department of Archaeology and Anthropology, University of Cambridge, for making the soil thin sections, as well as Jeffery Boyle and Margaret Davies of the Department of Crop and Soils Sciences, Washington State University, for their assistance with the soil fertility analyses.

\section{References}

Beach, T., Luzzadder-Beach, S., Cook, D., Dunning, N., Kennett, D.J., Krause, S., Terry, R., Trein, D., Valdez, F., 2015. Ancient Maya impacts on the earth's surface: an early Anthropocene analog? Quat. Sci. Rev. 124, 1-30.

Bonavia, D., 1967-1968. Investigaciones Arqueológicas en el Mantaro Medio. Rev. Mus. Nac. 35, 211-294.

Bowman, R., 1988. A rapid method to determine total phosphorus in soils. Soil Sci. Soc. Am. J. 52, 1301-1304.

Branch, N.P., Kemp, R.A., Silva, B., Meddens, F.M., Williams, A., Kendall, A., Vivanco Pomacanchari, C., 2007. Testing the sustainability and sensitivity to climatic change of terrace agricultural systems in the Peruvian Andes: a pilot study. J. Archaeol. Sci. 34, 1-9.

Brooks, S.O., 1998. Prehispanic Agricultural Terraces in the Río Japo Basin, Colca Valley, Peru. Unpublished Doctoral Dissertation Thesis, University of Wisconsin.

Bryant, R.B., Galbraith, J.M., 2010. Incorporating anthropogenic processes in soil classification. Soil Classification: A Global Desk Reference.

Bueno Mendoza, A., 2003. El Tiwantinsuyu en Huaytará. Investigaciones Sociales VII, pp. 41-56.

Bullock, P., Fedoroff, N., Jongerius, A., Stoops, G., Tursina, T., 1985. Handbook for Soil Thin Section Description. Waine Research, Wolverhampton.

Chauca Iparraguirre, G., Lane, K., 2015. Informe Final: Proyecto de Investigación Arqueológica de la Cuenca de Ica [PIACI] - Temporada 2014. Dirección General De Patrimonio Arqueológico Inmueble, Ministerio de Cultura, Lima.

Contreras, D.A., 2010. Landscape and environment: insights from the prehispanic Central Andes. J. Archaeol. Res. 18, 241-288.

Corwin, D., Lesch, S., 2003. Application of soil electrical conductivity to precision agriculture. Agron. J. 95, 455-471.

Courty, M.A., Goldberg, P., Macphail, R., 1989. Soils and Micromorphology in Archaeology. Cambridge University Press, Cambridge.

Covey, R.A., 2006. How the Incas Built their Heartland: State Formation and the Innovation of Imperial Strategies in the Sacred Valley, Peru. University of Michigan Press, MI.

D'Altroy, T.N., 2003. The Incas. Blackwell Publishing Ltd, Malden, MA.

de la Torre, C., Burga, M., 1986. Andenes y Camellones en el Peru Andino: Historia, Presente y Futuro. CONCYTEC, Lima.

De la Vega, G., 1960. Comentarios reales de los Incas. Editorial Universo, Lima.

Deetz, J., Dethlefsen, E., 1963. Soil pH as a tool in archaeological site interpretation. Am. Antiq. 29, 242-243.

Denevan, W.M., 1992. The pristine myth: the landscape of the Americas in 1492. Ann. Assoc. Am. Geogr. 82, 369-385.

Denevan, W.M., 2001. Cultivated Landscapes of Native Amazonia and the Andes. Oxford University Press, Oxford.

Devesa-Rey, R., Díaz-Fierros, F. Barral, M.T., 2011. Assessment of enrichment factors and grain size influence on the metal distribution in riverbed sediments (Anllóns River, NW Spain). Environ. Monit. Assess. 179, 371-388.

Donkin, R.A., 1979. Agricultural Terracing in the Aboriginal New World. University of Arizona Press, Tucson.

Dwomo, O., Dedzoe, C., 2010. Oxisol (ferralsol) development in two agro-ecological zones of Ghana: a preliminary evaluation of some profiles. J. Sci. Technol. 30, 11-28.

Eash, N.S., 1989. Natural and ancient agricultural soils in the Colca Valley, Peru. Unpublished Masters Thesis Thesis, Iowa State University.

Eash, N.S., Sandor, J.A., 1995. Soil chronosequence and geomorphology in a semi-arid valley in the Andes of Southern Peru. Geoderma 65, 59-79.

El-Swaify, S., 1980. Physical and mechanical properties of Oxisols. Soils with variable charge. Springer, New York, pp. 303-324.

Farrington, I.S., 1980. The archaeology of irrigation canals, with special reference to Peru. World Archaeol. 11, 287-305. 
French, C., 2015. One River Project: Sangayaico and uplands of the Ica basin: geoarchaeological and micromorphological analyses. University of Cambridge, McBurney Geoarchaeology Laboratory, Division of Archaeology, Internal Report.

Gardi, C., Angelini, M., Barceló, S., Comerma, J., Cruz Gaistardo, C., Encina Rojas, A., Jones, A. Krasilnikov, P., Mendonça Santos Brefin, M.L., Montanarella, L., Muñiz Ugarte, O., Schad, P., Vara Rodríguez, M.I., Vargas, R., Ravina da Silva, A.M.A. (Eds.), 2015. Soil Atlas of Latin America and the Caribbean. Publications Office of the EuropeanUnion, Luxembourg.

Gee, G., Bauder, J., 1986. Particle-Size Analysis. In: Klute, A. (Ed.), Methods of Soil Analysis: Part 1 - Physical and Mineralogical Methods. American Society of Agronomy, Madison, pp. 383-411.

Gee, G.W., Or, D., 2002. Particle-size analysis. In: Dane, J.H., Topp, G.C. (Eds.), Methods of Soil Analysis, Part 4. Physical Methods. American Society of Agronomy, Madison, pp. 255-293.

Gelles, P.H., 2000. Water and Power in Highland Peru: The Cultural Politics of Irrigation and Development. Rutgers University Press, New Brunswick.

Goodman-Elgar, M., 2002. Anthropogenic Landscapes of the Andes: A Multidisciplinary Approach to Precolumbian Agricultural Terraces and their Sustainable Use. University of Cambridge.

Goodman-Elgar, M., 2008. Evaluating soil resilience in long-term cultivation: a study of pre-Columbian terraces from the Paca Valley, Peru. J. Archaeol. Sci. 35, 3072-3086.

Goodman-Elgar, M., 2009. Places to Partake: Chicha in the Andean Landscape, Drink, Power, and Society in the Andes University Press of Florida, FL, pp. 75-107.

Guillet, D., 1981. Land tenure, ecological zone, and agricultural regime in the Central Andes. Am. Ethnol. 8, 139-156.

Guillet, D., 1987. On the potential for intensification of agropastoralism in the arid-zones of the Central Andes. In: Browman, D.L. (Ed.), Arid Land Use Strategies and Risk Management in the Andes: a Regional Anthropological Perspective. Westview Press, Boulder, pp. 81-98.

Hastorf, C.A., 1993. Agriculture and the onset of political inequality before the Inca. CUP Archive.

Heiri, O., Lotter, A.F., Lemcke, G., 2001. Loss on ignition as a method for estimating organic and carbonate content in sediments: reproducibility and comparability of results. J. Paleolimnol. 25, 101-110.

Holliday, V.T., Gartner, W.G., 2007. Soil phosphorus and archaeology: a review and comparison of methods. J. Archaeol. Sci. 34, 301-333.

Homburg, J.A., Sandor, J.A., Norton, J.B., 2005. Anthropogenic influences on Zuni agricultural soils. Geoarchaeology 20, 661-693.

Huaman Oros, O., Lane, K., 2014. Informe Final: Proyecto de Investigación Arqueológica de la Cuenca de Ica [PIACI] - Temporada 2013. Dirección General De Patrimonio Arqueológico Inmueble, Ministerio de Cultura, Lima.

Keller, J.M., Gee, G.W., 2006. Comparison of American Society of Testing Materials and Soil Science Society of America hydrometer methods for particle-size analysis. Soil Sci. Soc. Am. J. 70, 1094.

Kemp, R., Branch, N., Silva, B., Meddens, F., Williams, Kendall, A., Vivanco, C., 2006 Pedosedimentary, cultural and environmental significance of paleosols within prehispanic agricultural terraces in the southern Peruvian Andes. Quat. Int. 158, 13-22.

Kendall, A., Chepstow-Lusty, A., 2006. Cultural and environmental change in the Cuzco region of Peru: rural development implications of combined archaeological and paleoecological evidence. In: Dransart, P.Z. (Ed.), Kay Pacha: Cultivating Earth and Water in the Andes. John \& Erica Hedges Ltd, Oxford, pp. 185-197.

Konert, M., Vandenberghe, J., 1997. Comparison of laser grain size analysis with pipette and sieve analysis: a solution for the underestimation of the clay fraction. Sedimentology 44, 523-535.

Kosok, P., 1965. Life, Land, and Water in Ancient Peru: An Account of the Discovery, Exploration, and Mapping of Ancient Pyramids, Canals, Roads, Towns, Walls, and Fortresses of Coastal Peru with Observations of Various Aspects of Peruvian Life, Both Ancient and Modern. Long Island University Press, New York.

Kuhn, P., Aguliar, J., Miedema, R., 2010a. Textural Pedofeatures and Related Horizons. In: Stoops, G., Marcelino, V., Mees, F. (Eds.), Interpretation of micromorphological features of soils and regoliths. Elsevier, Amsterdam, pp. 217-250.

Kuo, S., 1996. Phosphorus. In: Sparks, D.L., Page, A.L., Helmke, P.A., Loeppert, R.H., Soltanpour, P.N., Tabatabai, M.A., Johnston, C.T., Sumner, M.E. (Eds.), Methods of Chemical Analysis. Part 3. Chemical Methods. Soil Science Society of America, Inc. Madison, WI, pp. 869-919.

Lane, K., 2006. Through the looking glass: re-assessing the role of agro-pastoralism in the north-central Andean highlands. World Archaeology 38, 493-510.

Lane, K., Oros, O.H., Beresford-Jones, D., 2015. Ritual y abandono en la Cuenca Alta Del Río Ica: el caso de Viejo Sangayaico [SAN 1]. Actas del I Congreso Nacional de Arqueología, 2014. Ministerio de Cultura, Lima.

Lentz, D.L., 2000. Imperfect balance: landscape transformations in the Precolumbian Americas. Columbia University Press, New York.

Londoño, A.C., 2008. Pattern and rate of erosion inferred from Inca agricultural terraces in arid southern Peru. Geomorphology 99, 13-25.

Maldonado Pimentel, A., Estacio Tamayo, V.A., 2012. Las primeras Mitas de Apurímac al servicio de las Minas de Castrovirreyna 1,591-1 and 599. Maldonado Pimentel, Lima.

Meurisse, R.T., Robbie, W.A., Niehoff, J., Ford, G., 1990. Dominant soil formation processes and properties in western-montane forest types and landscapes-some implications for productivity and management. Proceedings-Management and Productivity of Western-Montane forest soils, Boise, ID, pp. 7-19.

Mitchell, W.P., Guillet, D., 1994. Irrigation at high altitudes: the social organization of water control systems in the Andes. In: Ehrenreich, J.D. (Ed.), Society for Latin American Anthropology Publication Series. American Anthropological Association, Washington.
Montgomery, D.R., Balco, G., Willett, S.D., 2001. Climate, tectonics, and the morphology of the Andes. Geology 29, 579-582.

Mulvaney, R., 1996. Nitrogen-inorganic forms. In: Sparks, D.L., Page, A.L., Helmke, P.A., Loeppert, R.H. (Eds.), Methods of Chemical Analysis. Part 3. American Society of Agronomy, Madison, pp. 1132-1184.

Murphy, C.P., 1986. Thin Section Preparation of Soils and Sediments. AB Academic, Berkhamsted.

Nanavati, W., Sullivan, R., Bettencourt, N., Fortin, L., Goodman-Elgar, M., 2013. Characterizing tropical anthrosols by laser diffraction particle size analysis. 78th Annual Society for American Archaeology Meeting, Honolulu, HI.

Nettleton, W., Flach, K., Nelson, R., 1970. Pedogenic weathering of tonalite in southern California. Geoderma 4, 387-402.

Norton, J.B., Sandor, J.A., White, C.S., 2003. Hillslope soils and organic matter dynamics within a native American agroecosystem on the Colorado Plateau. Soil Sci. Soc. Am. J. 67, 225-234.

Olsen, S., Sommers, L., 1982. Phosphorus. In: Page, A., Miller, R., Keeney, D. (Eds.), Methods of Soil Analysis, Part 2. Soil Science Society of America, Madison, pp. 403-427.

Palacios Moncayo, O., 1994. In: Instituto Geológico, M.y.M. (Ed.), Geología de los Cuadrángulos de Santiago de Chocorvos y Paras. Carta Geológica Nacional, Lima, Perú.

Patriquin, D., 2003. Water, Soil and Organic Matter: a Complex Relationship. Dalhousie University, Halifax.

Pierzynski, G.M., Sims, J.T., Vance, G.F., 2005. Soils and Environmental Quality. CRC press, London.

Pulgar Vidal, J., 1946. Historia y Geografía del Perú. Universidad Nacional de San Marcos, Lima.

Rhoades, J., Chanduvi, F., Lesch, S., 1999. Soil Salinity Assessment: Methods and Interpretation of Electrical Conductivity Measurements. FAO, Rome.

Sandor, J.A., Eash, N., 1991. Significance of ancient agricultural soils for long-term agronomic studies and sustainable agriculture research. Agron. J. 83, 29-37.

Sandor, J.A., Eash, N., 1995. Ancient agricultural soils in the Andes of southern Peru. Soil Sci. Soc. Am. J. 59, 170-179.

Sandor, J.A., Furbee, L., 1996. Indigenous knowledge and classification of soils in the Andes of Southern Peru. Soil Sci. Soc. Am. J. 60, 1502-1512.

Sandor, J.A., et al., 2007. Biogeochemical studies of a native American runoff agroecosystem. Geoarchaeology 22, 359-386.

Schjellerup, I., 1986. Andenes y Camellones en la región de Chachapoyas. In: de la Torre, C., Burga, M. (Eds.), Andenes y Camellones en el Peru Andino: Historia. Presente y Futuro. CONCYTEC, Lima, pp. 133-150.

Schumacher, B.A., 2002. Methods for the determination of total organic carbon (TOC) in soils and sediments. Ecological Risk Assessment Support Center, pp. 1-23.

Silva, J.H., Deenik, J.L., Yost, R.S., Bruland, G.L., Crow, S.E., 2015. Improving clay conten measurement in oxidic and volcanic ash soils of Hawaii by increasing dispersant concentration and ultrasonic energy levels. Geoderma 237, 211-223.

Smith, J.L., Doran, J.W., Jones, A., 1996. Measurement and use of pH and electrical conductivity for soil quality analysis. In: Doran, J.W., Jones, A.J. (Eds.), Methods for Assessing Soil Quality. Soil Science Society of America, Madison, pp. 169-185.

Soil Survey Staff, 1993. Soil Survey Manual United States Department of Agriculture.

Stolt, M.H., Lindbo, D.L., 2010. Soil organic matter. In: Stoops, G., Marcelino, V., Mees, F. (Eds.), Interpretation of Micromorphological Features of Soils and Regoliths. Elsevier Amsterdam, pp. 369-396.

Stoops, G., 2003. Guidelines for Analysis and Description of Soil and Regolith Thin Sections. Soil Science Society of America, Madison.

Stoops, G., Marcelino, V., Mees, F. (Eds.), 2010. Interpretation of Micromorphological Features of Soils and Regoliths. Elsevier, Amsterdam.

Thomas, G., 1996. Soil pH and soil acidity. In: Sparks, D.L., Page, A.L., Helmke, P.A. Loeppert, R.H. (Eds.), Methods of Chemical AnalysisPart 3. American Society of Agronomy, Madison, pp. 475-490.

Thomaz, E.L., Antoneli, V., Doerr, S.H., 2014. Effects of fire on the physicochemical properties of soil in a slash-and-burn agriculture. catena 122, 209-215.

Trawick, P.B., 2003. The Struggle for Water in Peru: Comedy and Tragedy in the Andean Commons. Stanford University Press, Stanford.

Treacy, J.M., 1994. Las Chacras de Coporaque. Instituto de Estudios Peruanos, Lima.

Van Vliet-Lanoe, B., 2010. Frost action. In: Stoops, G., Marcelino, V., Mees, F. (Eds.), Interpretation of micromorphological features of soils and regoliths. Elsevier, Amsterdam, pp. 81-108.

W.R.B., 2014. World Reference Base for Soil Resources. World Soil Resources Report No. 106. F.A.O., Rome.

Wells, E.C., 2006. Cultural soilscapes. Geol. Soc. Lond., Spec. Publ. 266, 125-132.

Wernke, S.A., 2010. A reduced landscape: toward a multi-causal understanding of historic period agricultural deintensification in highland Peru. J. Lat. Am. Geogr. 9, 51-83.

Williams, P.R., 2006. Agricultural innovation, intensification, and sociopolitical development: the case of highland irrigation agriculture on the Pacific Andean watersheds. In: Marcus, J., Stanish, C. (Eds.), Agricultural Strategies. Cotsen Intitute of Archaeology, University of California, Los Angeles, pp. 309-333.

Zimmerer, K.S., 1991. Labor shortages and crop diversity in the southern Peruvian sierra. Geogr. Rev. 81, 414-432.

Zimmerer, K.S., 1998. The ecogeography of Andean potatoes. Bioscience 48, 445-454.

Zimmerer, K.S., 1999. Overlapping patchworks of mountain agriculture in Peru and Bolivia: toward a regional-global landscape model. Hum. Ecol. 27, 135-165. 